\title{
A comparison between X-shooter spectra and PHOENIX models across the HR-diagram
}

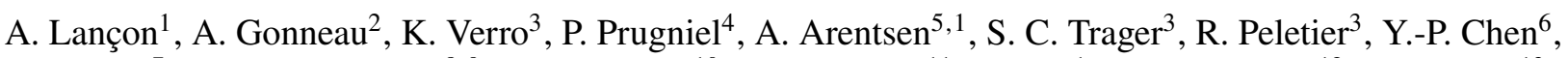 \\ P. Coelho ${ }^{7}$, J. Falcón-Barroso ${ }^{8,9}$, P. Hauschildt ${ }^{10}$, T.-O. Husser ${ }^{11}$, R. Jain ${ }^{1}$, M. Lyubenova ${ }^{12}$, L. Martins ${ }^{13}$, \\ P. Sánchez Blázquez ${ }^{14}$, and A. Vazdekis ${ }^{8,9}$
}

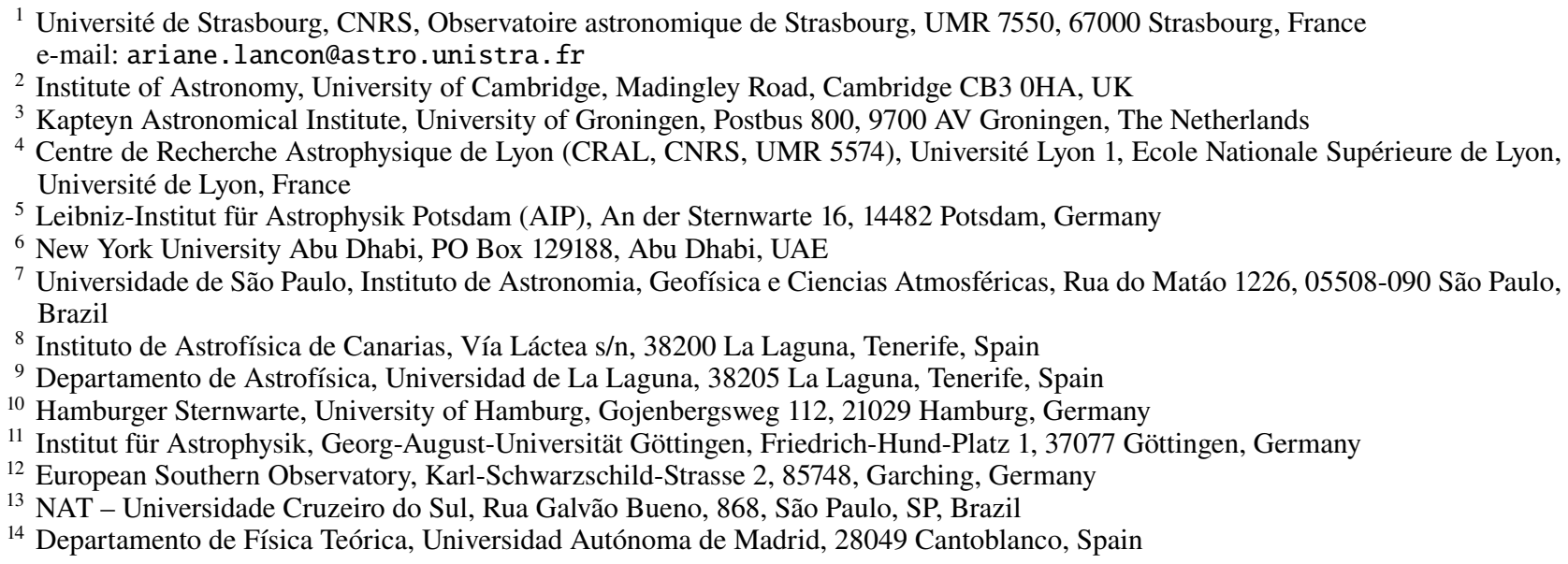

Received 8 September 2020 / Accepted 24 November 2020

\begin{abstract}
Aims. The path towards robust near-infrared extensions of stellar population models involves the confrontation between empirical and synthetic stellar spectral libraries across the wavelength ranges of photospheric emission. Indeed, the theory of stellar emission enters all population synthesis models, even when this is only implicit in the association of fundamental stellar parameters with empirical spectral library stars. With its near-ultraviolet to near-infrared coverage, the X-shooter Spectral Library (XSL) allows us to examine to what extent models succeed in reproducing stellar energy distributions (SEDs) and stellar absorption line spectra simultaneously. Methods. As a first example, this study compares the stellar spectra of XSL with those of the Göttingen Spectral Library, which are based on the PHOENIX synthesis code. The comparison was carried out both separately in the three arms of the X-shooter spectrograph known as UVB, VIS and NIR, and jointly across the whole spectrum. We did not discard the continuum in these comparisons; only reddening was allowed to modify the SEDs of the models.

Results. When adopting the stellar parameters published with data release DR2 of XSL, we find that the SEDs of the models are consistent with those of the data at temperatures above $5000 \mathrm{~K}$. Below $5000 \mathrm{~K}$, there are significant discrepancies in the SEDs. When leaving the stellar parameters free to adjust, satisfactory representations of the SEDs are obtained down to about $4000 \mathrm{~K}$. However, in particular below $5000 \mathrm{~K}$ and in the UVB spectral range, strong local residuals associated with intermediate resolution spectral features are then seen; the necessity of a compromise between reproducing the line spectra and reproducing the SEDs leads to dispersion between the parameters favored by various spectral ranges. We describe the main trends observed and we point out localized offsets between the parameters preferred in this global fit to the SEDs and the parameters in DR2. These depend in a complex way on the position in the Hertzsprung-Russell diagram (HRD). We estimate the effect of the offsets on bolometric corrections as a function of position in the HRD and use this for a brief discussion of their impact on the studies of stellar populations. A review of the literature shows that comparable discrepancies are mentioned in studies using other theoretical and empirical libraries.
\end{abstract}

Key words. stars: general - galaxies: stellar content - stars: atmospheres

\section{Introduction}

Stellar population studies based on the integrated spectra or colors of galaxies have a long history. Together with the studies of nearby resolved populations, they have allowed us to reach a broad understanding of the stellar contents of galaxies of different types or environments and of their components. While it may not be critical to characterize each galaxy in detail to study the average evolution of the stellar mass across cosmic time (e.g.,
Madau \& Dickinson 2014; Moutard et al. 2016), age and metallicity estimates become essential when studying the assembly of galaxy components; accurate interpretations of colors and spectral features are necessary to disentangle otherwise degenerate properties such as age, metallicity, and extinction, to obtain absolute rather than relative values for these quantities, or to constrain the stellar initial mass function based on integrated light. Such detailed investigations require population synthesis models to rest on robust stellar evolution calculations and on libraries 
of stellar spectra with both accurate spectral features and accurate spectral energy distributions (SEDs). Indeed, the bolometric corrections associated with the SEDs determine the relative contributions of any type of star at any particular wavelength, and these contributions are key ingredients of the interpretation of the summed spectral features of the stellar population under scrutiny.

In this context, it is disturbing that it remains so difficult to combine optical and near-infrared (near-IR) studies of stellar populations. Attempts to match the colors of large samples of galaxies from the near-ultraviolet (near-UV) to the near-IR have been found to leave significant residuals, sometimes leading authors to discard near-IR data in part of their analysis (Taylor et al. 2011). Star formation histories derived from optical and near-IR data separately, or from a given data set with different population synthesis models, still differ significantly (Powalka et al. 2017; Baldwin et al. 2018; Dahmer-Hahn et al. 2018; Dametto et al. 2019; Riffel et al. 2019). A part of the problem certainly lies in the theoretical modeling of advanced phases of stellar evolution, but it is also worth questioning the stellar spectral libraries. Indeed, if the balance between optical and near-infrared flux, for a given pattern of spectral features, is incorrect for individual stars, biases in the population synthesis models are inevitable.

Stellar spectral libraries can be either theoretical or empirical. In an ideal world, both would be equivalent. But this is a far-off target, and we are in the middle of a slow converging process that implies progress on both sides. Indeed, theoretical libraries cannot be tested without extensive empirical libraries, and empirical libraries cannot be used without stellar parameters, which are themselves estimated via comparisons with theoretical spectral properties. For the purpose of checking the overall consistency of spectral features and SEDs, continuity across the wavelength range of photospheric emission, a reasonable spectral resolution and a good spectrophotometric calibration are keys.

The above requirements can be fulfilled with instruments such as the X-shooter spectrograph on the Very Large Telescope of the European Southern Observatory. The three arms of $\mathrm{X}$-shooter, known as UVB, VIS, and NIR, together cover wavelengths from the near-UV well into the near-IR. The instrument was used to construct the X-shooter Spectral Library (XSL; Chen et al. 2014; Gonneau et al. 2020, hereafter Paper I), with the dual purpose of testing synthetic spectral libraries and serving as a direct ingredient for stellar population modeling. In this article, we provide the results of a first confrontation of these spectra with an extensive collection of synthetic spectra, namely the Göttingen Spectral Library (GSL; Husser et al. 2013). Comparisons with a few other model sets will follow and we encourage model-builders to repeat similar studies independently. Subsequent papers will present population synthesis models based on XSL, as well as the de-reddened, merged UVB, VIS, and NIR spectra constructed for these (Verro et al., in prep.).

Our immediate aims are (i) to investigate to what extent stellar parameters based on optical absorption line spectra (Arentsen et al. 2019, hereafter Paper II) lead to good matches between theoretical and empirical energy distributions over the wavelength range of X-shooter data and (ii) to determine to what extent the models are able to account for the global energy distribution of the data when the assumption that the parameters are those of Paper II is relaxed. Side products of this study are (iii) a comparison between the parameters obtained from individual spectral ranges (i.e., the UVB, VIS and NIR arms of X-shooter), (iv) a validation of the relative flux calibration of the X-shooter data, and (v) a quantitative estimate of the errors on bolometric corrections that may result from inconsistencies between SED-based and line-based parameter-estimates.

Considering the challenges of the modeling of cool stars, we expect to find the largest discrepancies between empirical and theoretical spectral energy distributions at low effective temperatures. For the practical purpose of population synthesis, any such discrepancies translate into uncertainties in the fundamental parameters associated with the empirical spectra. Issues that can be neglected to some extent when dealing with star and galaxy spectra in only a restricted spectral range, or with only low resolution SEDs, become more important to quantify when flux-calibrated spectra are used across optical to near-infrared wavelengths.

The main features of the GSL collection of synthetic spectra are recalled in Sect. 2 and those of the XSL data in Sect. 3. We then specify the two methods used to confront the empirical and theoretical data sets with each other in Sect. 4, and describe the results as a function of the position in the HR diagram in Sect. 5. For reasons that will become clear later, we focus on the most significant trends and on the temperature regime between 4000 and $5000 \mathrm{~K}$. Section 6 provides a comparison with other relatively recent confrontations between empirical and theoretical stellar spectra, a review of the limitations of GSL (many of which are common to a number of synthetic spectral libraries), and a brief discussion of the potential impacts of the trends we found, via bolometric corrections. The appendices provide a selection of additional figures; XSL-GSL comparison-figures for all the XSL spectra can be requested from the first author.

\section{The models}

The synthetic spectra used in this article are taken from Göttingen Spectral Library (GSL, Husser et al. 2013) in its version v $2^{1}$. The comparison with the X-shooter Spectral Library was one of the motivations for the computation of that grid, which provides a dense coverage of the HR-diagram at several compositions and covers the near-UV to near-IR range of X-shooter spectra at an adequate spectral resolution. This series of synthetic spectra provides a good representation of broad-band color-color relations of stars in the Milky Way (Figs. 2 and 22 of Powalka et al. 2016); it also provide good matches to optical absorption line spectra at medium resolution (e.g., Roth et al. 2018; Husser et al. 2016).

The underlying model atmospheres are obtained with version v16 of the PHOENIX code (Hauschildt et al. 1999), in spherical symmetry. This geometry is important in the low-gravity regime, where an atmosphere's extension is not negligible compared to the stellar radius (Scholz 1985; Plez et al. 1992; Heiter \& Eriksson 2006). The models and synthetic spectra adopt the equation of state and opacities referred to as PHOENIX-ACES, the calculation of which includes measured and theoretical lines lists by R. Kurucz as available in 2009 (>88 million metal lines and $>1$ billion molecular lines). PHOENIX models with these inputs were found to have a structure similar to that of MARCS models of the same period (see Sect. 7 of Gustafsson et al. 2008). Like many other large grids in the literature, the model spectra assume Local Thermal Equilibrium (LTE), which is a recognized limitation (Short \& Hauschildt 2003; Lanz \& Hubeny 2007).

The synthetic spectra are available on a grid of parameter space of which the four axes are the effective temperature $\left(T_{\text {eff }}\right)$, the surface gravity $\log (g)\left(g\right.$ in $\left.\mathrm{cm} \mathrm{s}^{-2}\right)$, the metallicity $[\mathrm{Fe} / \mathrm{H}]$ (with respect to the solar abundances of Asplund et al. 2009), and

http://phoenix.astro.physik. uni-goettingen.de/ 
Table 1. Parameter coverage of the GSL grid of synthetic spectra.

\begin{tabular}{lccl}
\hline \hline Variable & Range & Step size & \\
\hline$T_{\text {eff }}(\mathrm{K})$ & $2300 \ldots 7000$ & 100 & \\
& $7000 \ldots 12000$ & 200 & \\
& $12000 \ldots 15000$ & 500 & \\
$\log (g)$ & $-0.5 \ldots+5$ & 0.5 & No $\leqslant 0$ at high $T_{\text {eff }}$ \\
{$[\mathrm{Fe} / \mathrm{H}]$} & $-4.0 \ldots-2.0$ & 1.0 & \\
& $-2.0 \ldots+1.0$ & 0.5 & \\
{$[\alpha / \mathrm{Fe}]$} & $-0.2 \ldots+0.6$ & 0.2 & Full grid only at \\
& & & {$[\alpha / \mathrm{Fe}]=0$ and +0.6} \\
\hline
\end{tabular}

the $\alpha$-element enhancement $[\alpha / \mathrm{Fe}]$. The boundaries and sampling steps are summarized in Table 1 ; we note that a few $[\alpha / \mathrm{Fe}]$ ratios higher than listed are available but were not considered here. $\alpha$-enhancements affect the abundances of $\mathrm{O}, \mathrm{Ne}, \mathrm{Mg}, \mathrm{Si}$, $\mathrm{S}, \mathrm{Ar}, \mathrm{Ca}$ and $\mathrm{Ti}$. They are introduced at a given $[\mathrm{Fe} / \mathrm{H}]$, hence they affect the overall metallicity $Z$ of the models.

Spherical geometry introduces an extra degree of freedom compared to plane-parallel models. The adopted model masses are expressed as a simple function of $\log (g)$ and $T_{\text {eff }}$ (Fig. 1 of Husser et al. 2013); they range from about 0.5 to about $5 M_{\odot}$ along the main sequence, and reach values $>10 M_{\odot}$ at high temperatures and low gravities. Then surface gravity $g$ determines the radius. The stellar structures account for convection using the mixing length theory of turbulent transport, with a mixing length parameter in the range $[1,3.5]$ that depends on $T_{\text {eff }}$ and $\log (g)$.

Between 300 and $2500 \mathrm{~nm}$, the synthetic spectra are computed with a sampling step $\Delta \lambda$ such that $\lambda / \Delta \lambda \simeq 500000$. That such a high resolution is necessary for a good representation of empirical spectra of cool stars even at $R=3000$ has been known for some time (e.g., Fig. 1 of Lançon et al. 2007). The micro-turbulent velocities adopted to determine individual line profiles in the calculations are taken proportional to the average turbulent velocities of convection zones. They are typically around $1 \mathrm{~km} \mathrm{~s}^{-1}$ for $T_{\text {eff }}<9000 \mathrm{~K}$ and reach a maximum of about $3.5 \mathrm{~km} \mathrm{~s}^{-1}$ between 5000 and $6000 \mathrm{~K}$ at the lowest gravities (Fig. 3 of Husser et al. 2013). They drop to essentially zero above $10000 \mathrm{~K}$. The wavelengths of the synthetic spectra were converted from vacuum to air for comparison with the empirical spectra described below.

\section{The X-shooter spectra}

\subsection{The sample}

The X-shooter spectra used in this article are those of Data Release 2 of XSL (XSL-DR2, Paper II). Once carbon stars and incomplete spectra are excluded, the release contains data for 730 observations of 598 stars. Giants (on the red giant branch, on the asymptotic giant branch or in the red supergiant phase) represent $\sim 55 \%$ of these observations, dwarfs $\sim 35 \%$, and the remainder of the spectra belong to subgiants, to horizontal branch or blue loop stars, or in a few cases to objects in other short-lived transition phases.

Fundamental stellar parameters have been estimated for 713 of these spectra in Paper II. The effective temperatures range from $\sim 3500 \mathrm{~K}$ to $\sim 38000 \mathrm{~K}$ and the metallicities $[\mathrm{Fe} / \mathrm{H}]$ from $\sim-2.7$ to supersolar (Fig. 1). The 17 stars for which Arentsen et al. (2019) did not derive parameters are two late $\mathrm{M}$ dwarfs and some of the coolest Mira-type variables in XSL.

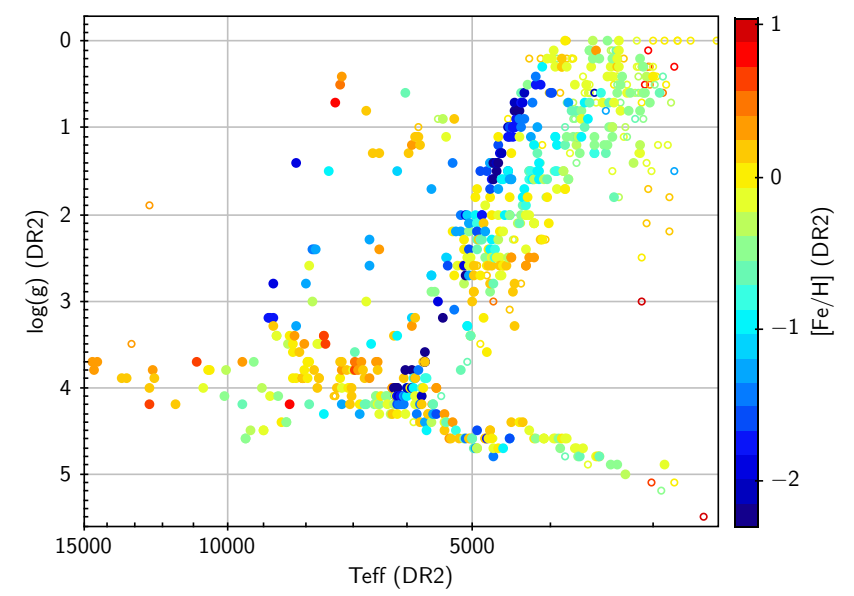

Fig. 1. Parameters of the XSL dataset, as evaluated by Arentsen et al. (2019). Open symbols correspond to spectra for which at least one of the three X-shooter arms was not corrected for slit-losses.

Gonneau et al. (2020) flagged a number of spectra as peculiar. We exclude three of these from the sample (X0214, X0248, X0424) because their energy distributions are obviously incompatible with the models used here: X0214 and X0248 correspond to an $\mathrm{Ae} / \mathrm{Be}$ star with a near-infrared excess due to circumstellar material, and X0424 to the combined emission of two stars. We further exclude the 6 hottest spectra of the remaining collection, whose estimated effective temperatures $(>21000 \mathrm{~K})$ are well beyond those available in the model grid.

In the end, we use a total of 704 spectra with parameter estimates from Paper II and we extend the sample to 721 spectra $(704+17)$ when the parameter estimates are not critical. We have not rejected long-period variable stars (LPVs) from our sample and figures, but they are clearly special cases for which we cannot expect good representations with synthetic spectra based on static atmosphere models (see Lançon et al. 2019, for preliminary results of a dedicated study). The results highlighted in this paper focus on regions of stellar parameter space where these stars have little or no impact.

\subsection{Properties of the spectra}

The properties most relevant to the analysis in this article are the wavelength coverage of the spectra, the spectral resolution, the flux calibration, and the signal-to-noise ratio $(\mathrm{S} / \mathrm{N})$. The XSLDR2 spectra are published in the rest-frame of each star, with wavelengths in air, and the exact wavelength coverage depends on the star observed. Before correction for radial velocity, the spectral ranges sampled by the UVB, VIS, and NIR arms of the X-shooter instrument are, respectively, 300-556, 533-1020, and 994-2480 nm. In practice, our analysis systematically excludes rest-frame wavelengths shorter than $400 \mathrm{~nm}$ for cool stars $\left(T_{\text {eff }}<\right.$ $4200 \mathrm{~K}$ ) and above $2.3 \mu \mathrm{m}$ for all stars; other masked regions are mentioned in Sect. 4.

As described in Paper $\mathrm{I}$, the resolving power $R=\lambda / \Delta \lambda$ is approximately constant across each arm, with $R \simeq 9800,11600$, and 8000 , respectively in the UVB, VIS, and NIR arms. Because this study focuses on spectral energy distributions and major spectral features, we choose to perform all comparisons at a constant, reduced resolving power of either $R=500$ or $R=3000$. This smoothing also has the advantage of reducing any sensitivity of the analysis to local residual wavelength calibration errors or variations in $R$. In practice, we first project the spectra onto an (oversampled) logarithmic wavelength scale and then convolve 
Table 2. Standard deviations of the color-differences between XSL and external datasets (from Gonneau et al. 2020).

\begin{tabular}{lccl}
\hline \hline $\begin{array}{l}\text { External } \\
\text { reference }\end{array}$ & Color & $\begin{array}{c}\text { Standard deviation } \\
\sigma(\mathrm{mag})\end{array}$ & Comments \\
\hline MILES & $(420-490)$ & 0.03 & 0.056 incl. 7 outliers \\
MILES & $(580-670)$ & 0.02 & 0.032 incl. 3 outliers \\
IRTF & $(J-H)$ & 0.023 & 0.033 incl. 6 outliers \\
IRTF & $\left(H-K_{s}\right)$ & 0.019 & 0.029 incl. 4 outliers \\
2MASS & $(J-H)$ & 0.032 & 0.050 incl. 2MASS errors \\
2MASS & $\left(H-K_{s}\right)$ & 0.038 & 0.056 incl. 2MASS errors \\
\hline
\end{tabular}

Notes. The synthetic colors in the first two lines are measured in 600 or $800 \mathrm{~nm}$-wide rectangular filters centered on the wavelengths indicated in the color-name, in nanometers. The 2MASS standard deviations listed each include six 4- $\sigma$ outliers. The outliers are not the same for all colors.

each arm with an adequate Gaussian broadening function. To reduce computation times, we project the empirical and synthetic spectra, after smoothing, to a regular log-wavelength scale with $\sim 3$ pixels per resolved element.

The XSL-DR2 data are available in two subsets, depending on whether or not a correction for slit-losses was applied to the fluxes. Among the 721 observations we consider, 597 are corrected for slit-losses in the UVB arm of X-shooter, 660 in the VIS arm, and 652 in the NIR arm. We focus on those spectra in our analysis. In Paper I synthetic broad band photometry was used to compare colors of the XSL spectra with those of the MILES and IRTF spectral libraries, and with colors in the 2MASS point-source catalog. For the colors measured, the distributions of the color-differences between the slit-loss corrected XSL spectra and other data typically have standard deviations of $\sim 0.03$ mag (Table 2 ), with a varying fraction of outliers (typically $2-7 \% 4-\sigma$ outliers). The systematic offsets compared to the MILES and IRTF colors are smaller than 1\%. The color-offsets with respect to 2MASS, also discussed in Paper I, are between 3 and $5.6 \%$, but these values depend sensitively on the selected subsample, on the modeled contribution of telluric absorption to the filter transmission functions, and also on the adopted Vega spectrum; they are compatible with 2MASS photometric errors and systematics in the near-infrared synthetic photometry.

The $\mathrm{S} / \mathrm{N}$ of the spectra are diverse, with typical values around 90 per original spectral pixel and above 100 after smoothing. We note that the computation of the initial noise spectra by the standard X-shooter pipeline, starting from the original 2-dimensional echelle images, does not keep track of correlations introduced by the rectification and wavelength calibration. This impacts the noise-propagation through subsequent transformations such as our smoothing procedure ${ }^{2}$. Also, we are aware of a number of cases in which the absolute level of the noise spectrum in one or the other X-shooter arm is dubious for unknown reasons (this is most obvious when the noise is overestimated). We must therefore interpret any result that depends on the absolute noise levels with care. However, the noise spectra do contain the signature of the blaze function of the spectrograph, they show the lower $\mathrm{S} / \mathrm{N}$

\footnotetext{
2 Some of the issues with X-shooter noise spectra are described for the UVB and VIS data in Schönebeck et al. (2014); the (partial) corrections suggested there are not implemented in the XSL pipeline, whose structure depends on the architecture of the original X-shooter instrument pipeline (distributed by the European Southern Observatory) until after the flatfielding and rectification of the spectra.
}

at both ends of the arm spectra, and they include a representation of the errors induced by the correction for telluric absorption (which is important in the near-infrared). Hence the inversevariance based on the noise spectra provides useful, though not statistically optimal, weights for the pixel-by-pixel comparison of a given XSL spectrum with several synthetic spectra.

\section{Data-model comparison methods}

Our data-model comparisons aim at evaluating in which regions of stellar parameter space the theoretical spectra are able to reproduce the (low or intermediate resolution) spectral energy distributions across the wavelength range of $\mathrm{X}$-shooter spectra. We do this first by adopting the stellar parameters of XSL-DR2 from Paper II as they are; then we repeat the exercise without the strong assumption that the parameters are known a priori.

An essential point to remember is that only optical absorption line spectra were exploited in Paper II: while applying the full-spectrum fitting code ULySS (Koleva et al. 2009), the authors discarded any information potentially carried by the SED, which was absorbed in a high order multiplicative polynomial of which the properties were not exploited. Discarding continuum information is a common procedure in stellar parameter estimation work and also in full-spectrum fitting methods for the analysis of the integrated light of stellar populations. Here, on the contrary, the low resolution energy distributions of the XSL spectra are an essential piece of information we examine. Hence our data-model comparisons do not allow for any polynomial corrections. Only extinction is allowed to modify energy distributions.

\subsection{Data-model comparison with the parameters of XSL-DR2}

The first data-model comparison is performed for the XSL spectra for which the three parameters $T_{\text {eff }}, \log (g)$, and $[\mathrm{Fe} / \mathrm{H}]$ were estimated in Paper II, and we adopt these values. As explained in detail in that article, these so-called "DR2-parameters" are tied to the estimated fundamental parameters of stars in previous empirical, optical spectral libraries (namely MILES and initially ELODIE; Prugniel \& Soubiran 2001; Sánchez-Blázquez et al. 2006), which are themselves the result of a detailed literature compilation followed by extensive work to homogenize values obtained by different methods and authors (Cenarro et al. 2007; Prugniel et al. 2011; Soubiran et al. 2016; Sharma et al. 2016).

Estimates of the $[\alpha / \mathrm{Fe}]$ ratios are not systematically available for the XSL stars and literature values are dispersed. After a first exploration using four values of $[\alpha / \mathrm{Fe}]$, we chose to base our analysis on synthetic spectra at $[\alpha / \mathrm{Fe}]=0$ and +0.4 , values that roughly represent the bulk of observed abundances in the Milky Way and Magellanic Clouds (Gonzalez et al. 2011; Nidever et al. 2020). The conclusions based on these two chemistries are similar, and the discussion further on explains why a more detailed approach would not be justified in this paper.

The discrepancies between the empirical and theoretical energy distributions are measured at low resolution $(R=500)$, using a normalized rms difference to quantify distance:

$D=\sqrt{\frac{1}{N_{\lambda}} \sum_{\lambda}\left(\frac{F_{\lambda}^{\mathrm{GSL}}-F_{\lambda}^{\mathrm{XSL}}}{F_{\lambda}^{\mathrm{XSL}}}\right)^{2}}$.

In this definition, $N_{\lambda}$ is the number of wavelengths accounted for in the sum, taking into account that a mask is used to exclude 
troublesome wavelength ranges (for instance regions with residuals from telluric absorption, ends of X-shooter arms that are affected by large flux calibration errors, and sometimes regions affected by line emission). $F^{\mathrm{XSL}}$ is the smoothed XSL spectrum and $F^{\mathrm{GSL}}$ is the smoothed, optimally reddened and optimally rescaled GSL spectrum under consideration. The extinction law of Cardelli et al. (1989) is used with a standard ratio of total to differential extinction, $R_{\mathrm{V}}=3.1$. For each GSL model, reddening is optimized by minimizing $D$ with respect to the extinction parameter $A_{\mathrm{V}}$. We allow only values of $A_{\mathrm{V}} \geqslant-0.1$, the negative values being tolerated to limit edge effects that could be due to flux calibration errors in the XSL data. For each $A_{\mathrm{V}}$, the optimal rescaling is re-evaluated.

In practice, the procedure is implemented in four variants that exploit four wavelength ranges. Each of the first three use only one of the X-shooter arms (UVB, VIS or NIR) to determine the best $A_{\mathrm{V}}$, while the fourth exploits all available wavelengths jointly (it is labeled ALL). In the fourth case, we allow the optimization procedure to select the rescaling factors for the UVB, VIS, and NIR ranges independently of each other. If the collection of model spectra contains a perfect match to the data, reddening included, then all the four variants of the adjustment procedure should point to the same $\mathrm{A}_{V}$.

For very cool or highly reddened stars, the flux drops to zero at the blue end of the UVB arm and flux calibration errors may even produce negative fluxes. To avoid that $D$ becomes exceedingly sensitive to regions of very low flux, we weight down the wavelengths ranges where the flux drops below $15 \%$ of the maximum flux of the arm.

The values of $D$ can be interpreted as typical fractional differences between the best reddened model and the data. Flux calibration errors with 1- $\sigma$ amplitudes equal to those listed in Col. 3 of Table 2, if they are assumed to correspond to simple errors in the general slope of the spectra of one X-shooter arm, translate into changes in $D$ of about 0.03 for each arm (with a slight dependence on the masked wavelength ranges used when computing $D$ ). Poor matches will be characterized by values of $D$ larger than $\sim 0.06$ as well as by inconsistent $\mathrm{A}_{V}$ between arms.

For the comparisons described in this section, we implemented an interpolation in the GSL grid before selecting the model nearest the parameters of a given XSL spectrum. The aim of the interpolation is to ensure that the discrepancy $D$ between neighboring models in the theoretical grid remains smaller than the $\sim 0.03$ that can result from flux calibration errors in the observations. Interpolation does not change the main trends discussed in this paper which, when we choose to mention them, are larger than a model grid step on average; but it reduces the dispersion due to finite sampling of parameter space and hence raises the level of significance of the trends. Our interpolation reduced the step in $T_{\text {eff }}$ by a factor of 2 (typically from $100 \mathrm{~K}$ to $50 \mathrm{~K}$ ), the step in $\log (g)$ by a factor of 2 (from 0.5 to 0.25 ), and the step in $[\mathrm{Fe} / \mathrm{H}]$ by a factor of 5 (from 0.5 to 0.1 , or at low metallicity from 1 to 0.2 ). We used spline interpolation, in a local volume around the target parameters typically including 27 original grid points at a given $[\alpha / \mathrm{Fe}]$. We did not interpolate in $[\alpha / \mathrm{Fe}]$.

\subsection{The search for best-fit models}

In the search for a best-fit model, $T_{\text {eff }}, \log (g),[\mathrm{Fe} / \mathrm{H}]$, and $[\alpha / \mathrm{Fe}]$ are free parameters and the DR2-values of Paper II are used only to initiate the optimization algorithm. Where useful, we include the 17 spectra with no associated parameters that were identified in Sect. 3.1, and we assign them initial-guess effective temperatures between 2300 and $3000 \mathrm{~K}$, a surface gravity $\log (g)=0$ or 4.5 , and a metallicity $[\mathrm{Fe} / \mathrm{H}]=0$ (based on spectral type).

At any given $[\alpha / \mathrm{Fe}]$, a subset of synthetic spectra centered on the DR2-parameters is selected. For each of these model spectra separately, a best-match extinction parameter $A_{\mathrm{V}}$ is obtained, using the standard extinction law of Cardelli et al. (1989) with $R_{\mathrm{V}}=3.1 . A_{\mathrm{V}}<-0.1$ is prohibited. The fit-quality is measured at resolution $R=500$ or $R=3000$ with a classical inverse-variance weighted $\chi^{2}$-difference between the reddened model and the XSL-spectrum, or alternatively with the quantity $D$ of the previous section (Eq. (1)). If the best-fitting reddened model lies on the edge of the subset of models first selected, the subset is extended in the adequate direction until this is not the case (except on edges of the available model grid).

As in the previous section, the fitting procedure is implemented in four variants that exploit four wavelength ranges (UVB, VIS, NIR, and ALL). If the collection of model spectra contains a perfect match to the data, reddening included, then all these variants point to the same set of parameters.

When the figure of merit for each arm is the inverse-variance weighted $\chi^{2}$, the fourth variant of the fitting procedure, which we refer to as the "global fit", uses a combined- $\chi^{2}$ based on the values obtained separately in each arm. There are numerous ways to proceed and we have implemented several, but since we restrict our discussion to major trends any choice that ensures a relatively even balance between the three arms leads to similar conclusions. The spectra of the three arms each contain comparable numbers of resolved spectral elements $\left(\sim 10^{4}\right)$ and we choose to give all three similar importance by calculating

$\chi_{\text {global }}^{2}=\frac{1}{3}\left(\frac{\chi_{\mathrm{UVB}}^{2}}{\min \left(\chi_{\mathrm{UVB}}^{2}\right)}+\frac{\chi_{\mathrm{VIS}}^{2}}{\min \left(\chi_{\mathrm{VIS}}^{2}\right)}+\frac{\chi_{\mathrm{NIR}}^{2}}{\min \left(\chi_{\mathrm{NIR}}^{2}\right)}\right)$,

where the minima are taken over the subset of all reddened models to which a given XSL spectrum was compared. To this latter purpose, the $\chi^{2}$-values per arm are initially saved for each synthetic spectrum in the subset and for a broad range of values $A_{\mathrm{V}}$ including the values found best in each arm; the $A_{\mathrm{V}}$ of the best global fit, like the other parameters, can differ from each of the by-arm values. An advantage of this choice of $\chi_{\text {global }}^{2}$ is its low sensitivity to errors in the scaling of the noise spectrum that may affect one or the other arm; a caveat is the excessive weight that might be attributed to a spectral arm with poorer $\mathrm{S} / \mathrm{N}$.

The interpolation scheme of the previous section is also implemented here. We recall however that our point is not to re-evaluate stellar parameters per se, but to determine where in the HR-diagram good matches are possible or otherwise what the main trends in the discrepancies are. These conclusions are already apparent without interpolating. The procedure above produces a number of figures for eye inspection, of which examples are provided in Appendix A.

\section{Results}

\subsection{The SEDs for the parameters of DR2}

The differences between empirical and theoretical energy distributions as measured by $D$ (Eq. (1)) display systematic trends as a function of effective temperature, surface gravity, and metallicity, and we have therefore chosen to present them in HR-diagrams directly comparable to Fig. 1. The maps for the four wavelength ranges we have considered (UVB, VIS, NIR, and ALL) are shown in Fig. 2. For comparison, the values of $D$ 

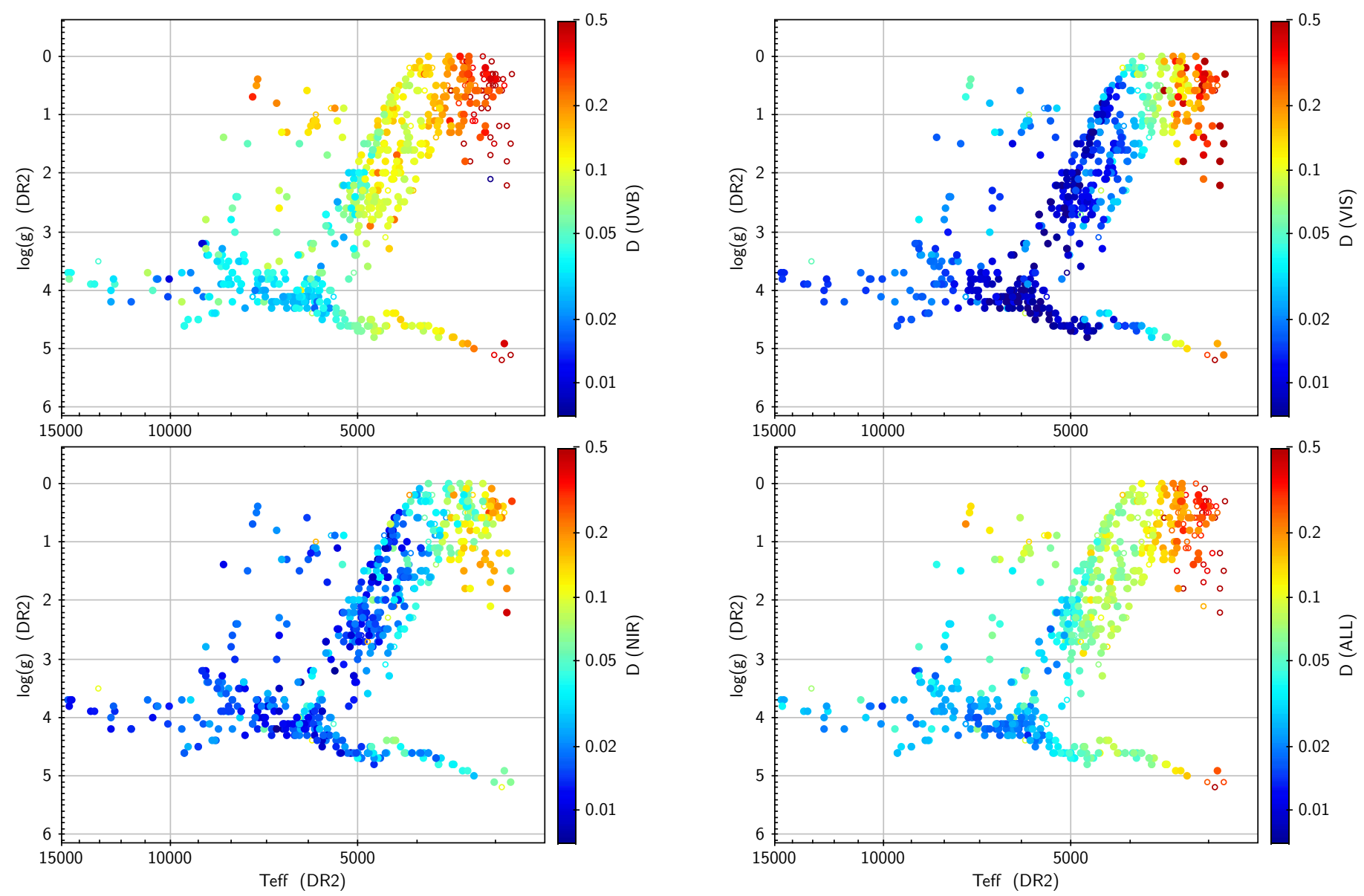

Fig. 2. Comparison between the low-resolution SEDs $(R=500)$ of XSL spectra and the nearest GSL spectrum, when adopting the fundamental parameters of Arentsen et al. (2019). Only extinction is allowed to modify the energy distribution of the theoretical spectra. The color-coding shows $D$ (Eq. (1)), as measured in the UVB, VIS, and NIR arms and across the whole spectrum. Open symbols correspond to spectra for which no slit-loss corrections were applied. $1-\sigma$ flux calibration errors correspond to $D \simeq 0.03$ in each of the UVB, VIS, and NIR arms.

resulting solely from the finite sampling of parameter space in the theoretical spectral library can be examined in Appendix B. A selection of direct comparisons of the XSL and GSL low resolution spectra, for the parameters of Paper II as adopted in this section, can be found in Appendix C.

Strong systematic trends dominate the aspect of the four panels of Fig. 2, with comparatively small local scatter. This provides evidence that we are indeed measuring differences between empirical and theoretical stellar energy distributions rather than any dominant random errors in the XSL SEDs that previous examination of these data would have missed, or random errors due to the finite sampling of the model grid.

In the VIS and NIR arms, the theoretical SEDs are in excellent agreement with the empirical SEDs over vast regions of the HR diagram, corresponding roughly to $T_{\text {eff }}>4000 \mathrm{~K}$. This is a remarkable achievement of the GSL collection. The consistently low values of $D$ over this part of parameter space $(D \leqslant 0.03)$ indicate that the photometric errors recalled in Table 2 are not underestimated.

In the UVB arm, the model SEDs for the upper main sequence $\left(T_{\text {eff }}>5000 \mathrm{~K}\right)$ also agree with the data, albeit with somewhat larger discrepancies $D$ than in the VIS and NIR. With respect to the standard deviation $\sigma$ associated with flux calibration errors, the discrepancies are distributed between essentially 0 and about $2-\sigma(D \simeq 0.06)$. However, below $\sim 5000 \mathrm{~K}$ the models disagree significantly with the UVB SEDs $(D>0.06)$ for essentially all stars. They also disagree for the warm low-gravity stars in XSL. As a consequence, the energy distributions across all wavelengths are also in disagreement for these regimes.

Before discussing the general trends further, we briefly examine the most extreme outliers in Fig. 2. Among the three data points near $T_{\text {eff }}=7500 \mathrm{~K}$ and $\log (g)=0.5$, the two lower gravity ones are two observations of the same stars, a high metallicity star with one of the highest extinction estimates in the sample $\left(A_{\mathrm{V}} \simeq 3\right)$; for the third data point, the literature and our own estimates in Sect. 5.2 suggest the $T_{\text {eff }}$ of Paper II is too high by more than $1500 \mathrm{~K}$ (the DR2-parameters had been correspondingly flagged). Between 4000 and $5000 \mathrm{~K}$, the spectra with the largest values of $D(\mathrm{UVB})$ or $D(\mathrm{ALL})$ are among those for which the observed fluxes are near zero below $400 \mathrm{~nm}$, and the exact definition of the denominator in Eq. (1) matters. This is one of our reasons for focusing on typical situations and general trends instead of individual objects in this article.

Below $4000 \mathrm{~K}$, it is notorious that stellar spectra are difficult to model, for instance because of large surface convection cells, of variability, of the formation of dust grains. In the following, we choose to concentrate on temperatures between 4000 and $5000 \mathrm{~K}$, where these issues are in principle less severe. This regime contains stars with strong contributions to the integrated light of stellar populations over a broad range of optical and near-infrared wavelengths, which is a further reason for our interest. 
The nature of the disagreement for stars between 4000 and $5000 \mathrm{~K}$ is illustrated with typical cases in Figs. C.5 to C.11. There are systematic differences in spectral features (in particular in the UVB range), but our focus here is on energy distributions. Whatever wavelength range is used to estimate $A_{\mathrm{V}}$, discontinuities appear in the figures at the limits between X-shooter's spectral arms, showing that the energy distributions are not matched properly. Moreover, the steps are in the same direction for all the spectra shown (the examples are selected to be representative, only rare cases deviate from this behavior). Let us for instance examine Figs. C.7 and C.11, where the extinction applied to the synthetic spectra is optimized using the VIS arm: the empirical spectra in the UVB and NIR fall off faster than the theoretical spectra when moving away from the VIS range. If the empirical UVB and NIR spectra were rescaled to connect to the VIS data smoothly, the merged spectrum would lack flux both in the UVB and NIR compared to the model. This trend is more obvious at low metallicities (Fig. C.7) than at near-solar metallicity (Fig. C.11).

Another manifestation of the mismatch between empirical and theoretical SEDs is seen when comparing the extinction estimates obtained separately from the UVB and the VIS data: Fig. 3 shows that below about $5000 \mathrm{~K}, A_{\mathrm{V}}$ (UVB) is in general larger than $A_{\mathrm{V}}$ (VIS). Both also tend to exceed $A_{\mathrm{V}}$ (NIR), but because of the wavelength dependence of extinction laws, $A_{\mathrm{V}}(\mathrm{NIR})$ is more sensitive to flux calibration errors and that trend is more dispersed. In brief: when using the UVB arm for the fit, a large $A_{\mathrm{V}}$ is preferred and the theoretical spectrum has excess flux in the NIR compared to the observations; when using the NIR arm for the fit, a small $A_{\mathrm{V}}$ is preferred and the theoretical spectrum has excess flux in the UVB compared to the observations.

Three hypotheses come to one's mind immediately: (i) either the extinction law is inadequate (it should rise more steeply towards the ultraviolet than the steepest law we explored), (ii) or the models lack opacities with a more severe deficit at shorter wavelengths, (iii) or the parameters in Paper II are systematically offset for some other reason from those that would provide good fits with the GSL library.

Proposition (i) is unlikely, considering what is known about extinction in the Local Universe (Schlafly et al. 2016). It would also imply a correlation between the difference $A_{\mathrm{V}}(\mathrm{UVB})-A_{\mathrm{V}}(\mathrm{VIS})$ and a mean estimate of extinction which, if present at all, is weak in our data. Propositions (ii) and (iii) on the other hand are reminiscent of previous studies with various collections of synthetic and empirical spectra. To test propositions (ii) and (iii) further, the results of the free search for the best-fit GSL match to each XSL observation are needed and we therefore postpone discussions to Sects. 5.2 and 6 .

We complete this part with a brief comment on the $[\alpha / \mathrm{Fe}]$ ratio. The results shown in Figs. 2 and 3 combine estimates obtained with $[\alpha / \mathrm{Fe}]=0$ and $[\alpha / \mathrm{Fe}]=+0.4$. Outside the range of parameters covered by the $\alpha$-enhanced models, that is for $T_{\text {eff }}>8000 \mathrm{~K}, T_{\text {eff }}<3500 \mathrm{~K}, \log (g)<-0.1$ or $[\mathrm{Fe} / \mathrm{H}]>+0.1$, solar $\alpha$-abundances were used. Elsewhere, we decided that the $\alpha$ enhanced model was favored over the solar one when that change in abundances reduced $D$ (UVB) by at least 0.01 , without degrading $D$ (VIS), $D$ (NIR), $D$ (ALL) or $\mid A_{\mathrm{V}}$ (UVB) $-A_{\mathrm{V}}$ (VIS) $\mid$ significantly. The XSL-spectra favoring $\alpha$-enhanced models are those of metal-poor giants, as expected from statistics in the Milky Way. Eye-inspection of the superimposed empirical and theoretical spectra with solar and with $\alpha$-enhanced abundances, be it at $R=500$ or $R=3000$, then confirms the better match of the $\mathrm{Ca}$ II lines or the Mg I triplet (which are deeper in $\alpha$-enhanced models) and the $\mathrm{CH}$ and $\mathrm{CN}$ bands (which are shallower in

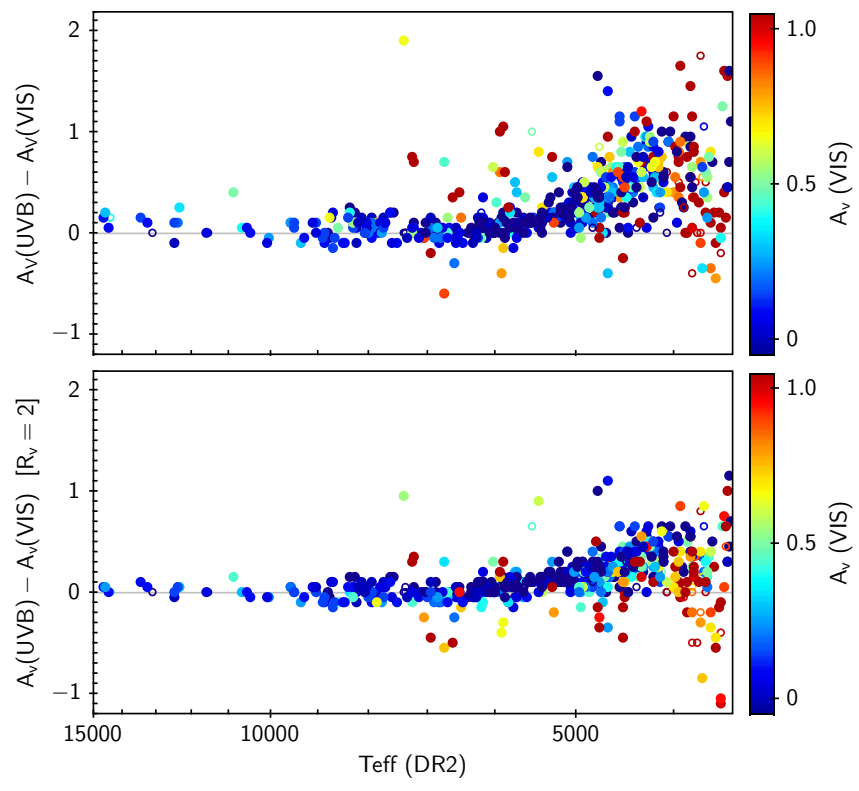

Fig. 3. Difference between the extinction estimates obtained with the UVB and VIS segments of the XSL data, from the comparison with GSL energy distributions when adopting the stellar parameters of Arentsen et al. (2019). Top: standard extinction law $(\mathrm{Rv}=3.1)$. Bottom: extreme extinction law $(\mathrm{Rv}=2)$.

$\alpha$-enhanced models because enhanced O captures a larger fraction of the available C). However, the effect of accounting for $\alpha$-enhancement produces only very small changes in Fig. 2, that the untrained eye would not even notice. The trends discussed above are unchanged.

In summary, the SEDs of the GSL models agree well with the SEDs of XSL, for the parameters of Paper II, over wide parts of the HR-diagram. But there is statistically significant systematic disagreement below $\sim 5000 \mathrm{~K}$, and for luminous warm stars (though with lower significance due to smaller numbers).

In the following section, we relax the assumption that the fundamental parameters are known and we search for the bestfitting model with that extra freedom. If models can be found that match the SEDs to within the errors (in the data and due to the discrete grid), we would be facing a classic parameter calibration problem: the reference libraries used in Paper II and GSL are different, and this may lead to offsets in derived parameters. It would remain to be determined which calibration is more robust. If adequate models cannot be found in certain parts of the HRdiagram, then these should be regions on which to focus future efforts in stellar spectral synthesis.

\subsection{Best-fit model SEDs for each XSL spectrum}

When the stellar parameters are free, better matches with GSL energy distributions can be obtained in the critical regions of the HR-diagram identified above. $D$ now takes values between 0.03 and 0.06 for effective temperatures between 4000 and $5000 \mathrm{~K}$ (Fig. 4). Such values, taken individually, would be consistent with flux calibration errors at the $2 \sigma$ level. In fact a part of the discrepancies are due to local spectral features, and hence the part of $D$ associated with the general low resolution energy distribution is even smaller than these $2 \sigma$.

However the homogeneous behavior of the numerous data points below $5000 \mathrm{~K}$ in Fig. 4 tells us that the values of $D$ are not the result of random errors. Systematic discrepancies are still present. Despite the free exploration of parameter space, we must 



Fig. 4. Top: change in the discrepancy measure $D$ (Eq. (1)) when, instead of adopting stellar parameters from Arentsen et al. (2019), the parameters are freely optimized. The data points are located in the diagram according to the DR2-parameters of Arentsen et al. (2019) The symbol color maps the difference between the best-fit value of $D$ (Eq. (1)) and its value for the DR2-parameters. Bottom: discrepancy $D$ for the best-fit stellar parameters (to be compared to the bottom right panel of Fig. 2, where the parameters of Arentsen et al. (2019) were assumed). Complete versions of these figures, with one panel per arm, are available in Figs. D.1 and D.2.

conclude that it remains difficult to reproduce both the spectral features and the low-resolution SEDs of the cool stars simultaneously below $5000 \mathrm{~K}$. Below, in Sect. 5.2.1, we clarify the nature of the systematic discrepancies seen between the models with the best-fitting SEDs and the observations, where such discrepancies are present. Subsequently, in Sect. 5.2.2, we quantify how the parameters of the best-SED models obtained here compare to those of Paper II.

\subsubsection{Residual differences between best-SED models and XSL spectra}

When the purpose is to find the best theoretical match to a given observation (as opposed to comparing the quality-of-fit for different observations), inverse-variance weighted $\chi^{2}$ values are a more appropriate figure of merit than $D$, which gives low-flux regions at short wavelengths too much weight. For the sake of validation, we have performed all calculations with the two methods. The directions of the trends are unchanged, but amplitudes of the differences between the parameters from optimization and the parameters in Paper II depend on the weighting, in the sense that slightly larger differences are found on average when using $D$, than when using the inverse-variance weighted $\chi^{2}$. In the following, we restrict the figures presented to those obtained with the weighted $\chi^{2}$. We focus on temperatures between 4000 and $5000 \mathrm{~K}$.

Examples of matched spectral energy distributions, based on fits performed at $R=500$ by minimizing the inverse-variance weighted $\chi^{2}$ across all available wavelengths (Eq. (2)), are shown in Figs. 5 and 6 respectively for relatively metal-poor and relatively metal-rich stars, with effective temperatures between 4000 and $5000 \mathrm{~K}$ according to Arentsen et al. (2019). The energy distributions from the near-UV to $2.4 \mu \mathrm{m}$ are reproduced well for the new sets of stellar parameters indicated in the panels (compare with Figs. C.5 and C.9). The residuals are roughly flat on average and the three arms in general connect naturally. In the near-IR, where the main low-resolution features are the shape of the $\mathrm{H}$-band (set by continuous $\mathrm{H}^{-}$opacities) and the $\mathrm{CN}$ band near $1.1 \mu \mathrm{m}$, excellent agreement with the observed shapes are obtained.

However, in this temperature range it is clear that the improvement in the SEDs does not always come with a good match of all the spectral features. The local features in the residuals are of varying amplitude but are generally highly significant (the $\mathrm{S} / \mathrm{N}$ per resolved element is of several hundred at $R=500$ ). They are not random but correspond to features in the spectra. There is some natural dispersion in the properties of the residuals and we cannot provide an exhaustive description; the points we mention are those for which we have enough cases to consider they are systematic trends.

A striking first impression is that the UVB residuals would not satisfy any expert of stellar parameter estimates. They are clearly larger than those one can expect to achieve when fitting spectra over the traditional optical range $(400-700 \mathrm{~nm})$ when allowing for a polynomial correction of the continuum. They are also significantly larger than those we obtain with GSL when fitting only the UVB range with reddened models, instead of ALL wavelengths. In the VIS and NIR ranges, the features are intrinsically weaker than in the UVB, and the physical natural variance is smaller between 4000 and $5000 \mathrm{~K}$. The tension between the SED and the spectral features is still present to some extent, but weaker.

In luminous metal-poor giants (top rows of each panel of Fig. 5), the best-SED models tend to display a deeper $G$-band (CH molecule, $0.55 \mu \mathrm{m}$ ) than the observations. A fit restricted to the UVB arm would be able to eliminate this issue, but at the cost of degrading the panchromatic SED. At warm enough temperatures in the range considered, the metal-poor models display hydrogen lines, which for the best-SED tend to be too weak in the Balmer series but too deep in the near-IR Brackett series.

At higher giant-branch gravities $(\log (g) \simeq 2)$, an interesting trend appears across the UVB spectrum of metal-poor giants (middle rows in the upper right panel of Fig. 5): the largest residuals take the shape of positive features at wavelengths longer than $0.49 \mu \mathrm{m}$, while it is the opposite below $0.43 \mu \mathrm{m}$. A closer look shows that the largest residual differences at the red end of the UVB arm correspond to strong metal lines, that are too deep in the best-SED models; this suggests that the constraints from the panchromatic SED pull the fit towards high metallicities. At the blue end of the UVB, the largest residuals are not associated with strong lines, but are more broadly distributed. For main sequence stars (last rows in the panels of Fig. 5), the largest residuals below $0.43 \mu \mathrm{m}$ correspond to regions in between the deepest spectral features.

Moving to higher metallicities (Fig. 6), the trends just described for gravities $\log (g) \gtrsim 2$ remain present. The multitude of lines of molecular and atomic species makes it difficult to 
A. Lançon et al.: A comparison between X-shooter spectra and PHOENIX models across the HR-diagram
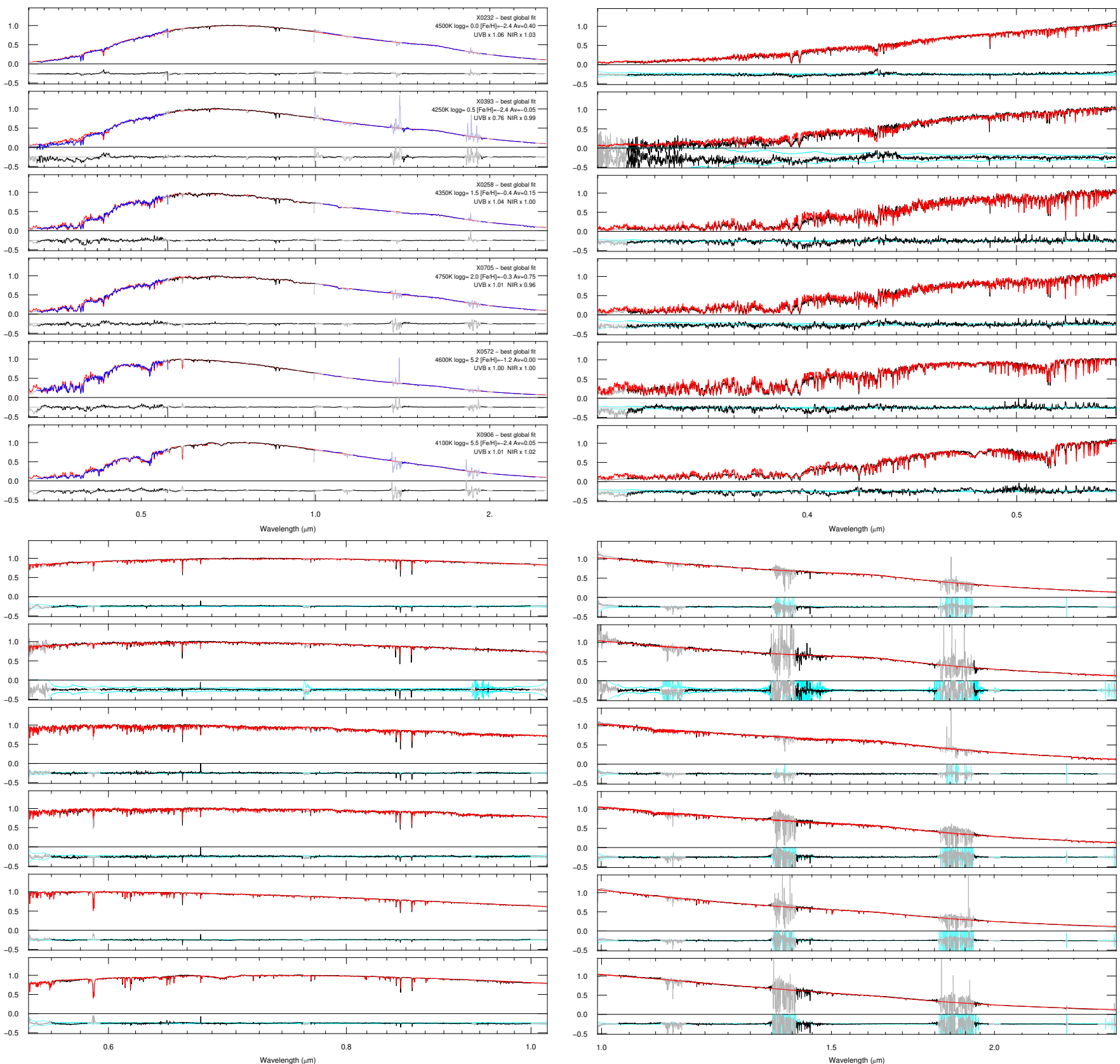

Fig. 5. Typical comparisons between XSL and best-match GSL energy distributions for stars with relatively low metallicities according to XSL DR2 and with DR2- $T_{\text {eff }}$ between 4000 and $5000 \mathrm{~K}$. The comparisons are shown for the parameters that minimize the combined inverse-variance weighted $\chi^{2}$ over all wavelengths (Eq. (2)). The upper left panels show all wavelengths at $R=500$, the other panels are zooms into these same comparisons, at $R=3000$. The best-match synthetic spectra are in red; the empirical spectra in black, except in the upper left panel where they are shown in blue, black, and blue for the UVB, VIS, and NIR arms of X-shooter. Below each spectrum, the residuals are shown together with positive and a negative version of the XSL error spectrum (cyan). Gravity increases from top to bottom: HD 165195 (X0232), HD 1638 (X0258), NGC 68381037 (X0705), LHS 1841 (X0572), LHS 343 (X0906). Compare with Fig. C.5.

emphasize any other feature specifically. By letting the eye slide over the residuals presented, it can be seen that their features repeat (within a given regime of the HR diagram). The discrepancies are mostly systematic, rather than random. The calcium triplet for instance, around $0.86 \mu \mathrm{m}$, is usually too strong in the best-SED model for giants (the SED favoring $[\alpha / \mathrm{Fe}]=0$ at solar-like metallicities), while it is well matched in the dwarfs.

The tension between the SED and the spectral features also manifests in the sensitivity of the best-fit parameters to the wavelength range considered in the comparison. For instance,
$\Delta A_{\mathrm{V}} \equiv A_{\mathrm{V}}(\mathrm{UVB})-A_{\mathrm{V}}(\mathrm{VIS})$ still is positive on average between 4000 and $5000 \mathrm{~K}$, with $80 \%$ of the values spread between 0 and 0.8 (Fig. 7; compare with Fig. 3). This apparent temperaturedependence of $\Delta A_{\mathrm{V}}$ is due primarily to mismatches in the UVB arm, where the density of strong spectral lines is largest. As described above, the simultaneous fit to all three arms produces residual features in the UVB with a systematic sign-difference between the blue end and the red end of that arm. A larger extinction in the UVB would help reduce these residuals, and this happens in fits that do not use the VIS and NIR constraints. On 

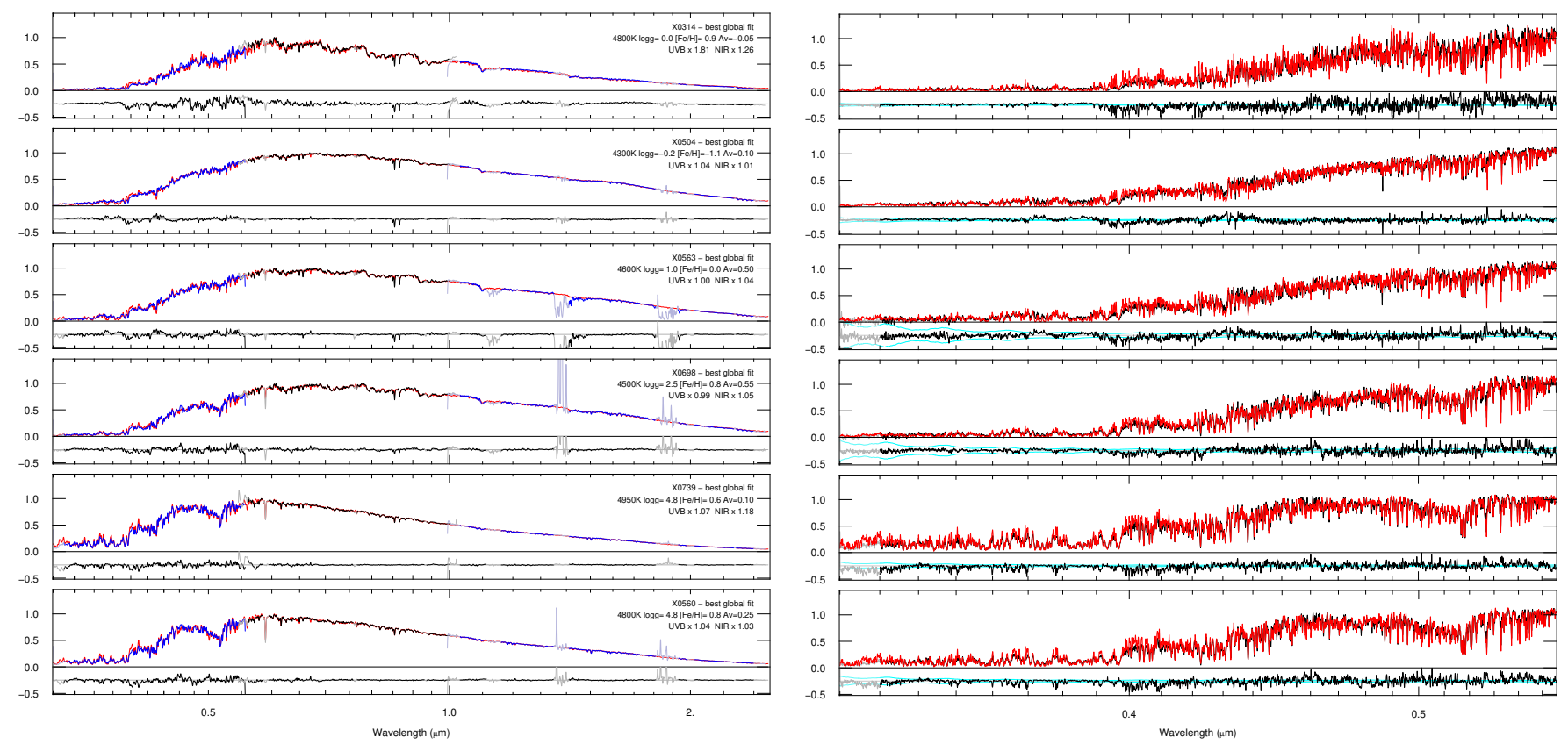

Fig. 6. Typical comparisons between XSL and best-match GSL energy distributions for stars with relatively high metallicities according to XSL DR2 and with DR2- $T_{\text {eff }}$ between 4000 and $5000 \mathrm{~K}$. The comparisons are shown at $R=500$, for the parameters that minimize the combined inversevariance weighted $\chi^{2}$ over all wavelengths (Eq. (2)). Gravity increases from top to bottom: HD 50877 (X0314), BBB SMC 104 (X0504), HD 44391 (X0563), 2MASS J18351420-3438060 (X0698), HD 218566 (X0739), HD 21197 (X0560). In the case of X0504, the best-match metallicity is lower than the DR2-value (-0.6), which had justified its presence is this subset of spectra. Compare with Fig. C.9.

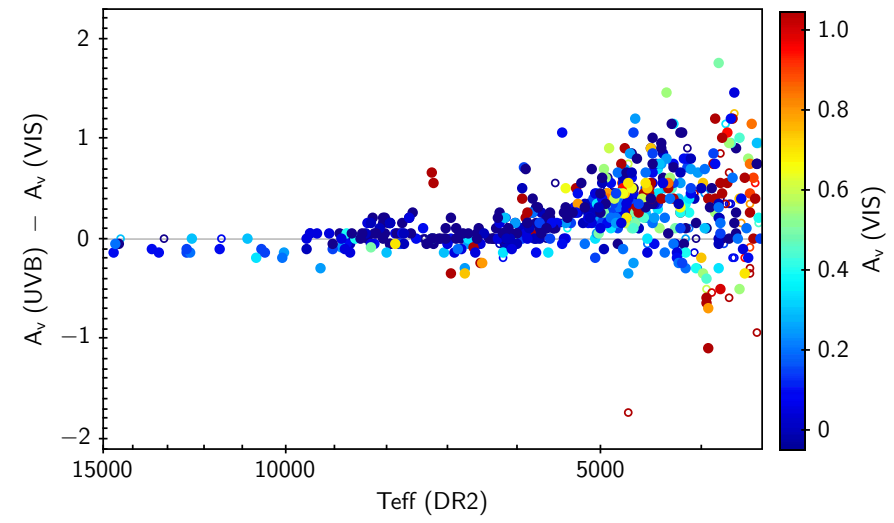

Fig. 7. Same as Fig. 3, but for the parameters and extinction that minimize the inverse-variance weighted $\chi^{2}$-difference between models and data in the UVB and VIS arms of X-shooter.

average, the extinction estimate based on the VIS arm compares well with the extinction evaluated from the three arms together.

In the comparison between parameters preferred by the different arms of the spectrograph, the effects of degeneracies are seen strongly. For the sample as a whole, the difference $\Delta A_{\mathrm{V}}$ correlates positively with the corresponding differences in $T_{\text {eff }}$ and in $[\mathrm{Fe} / \mathrm{H}]$ obtained using the UVB or the VIS arm, as expected from the notorious effects on $T_{\text {eff }}$ and $A_{\mathrm{V}}$ on spectral slopes, and from the need to compensate a higher $T_{\text {eff }}$ with higher metallicity in order to obtain spectral features of similar strength. However, this hides a more complex dependence on position in the HR diagram and on metallicity. A few examples are given in Appendix E.

We find no significant correlation between $\Delta A_{\mathrm{V}}$ and $A_{\mathrm{V}}(\mathrm{ALL})$ or $\left[A_{\mathrm{V}}(\mathrm{UVB})+A_{\mathrm{V}}(\mathrm{VIS})\right] / 2$ for the sample as a whole, which would have been the most evident indication of an inadequate extinction law. Nevertheless, we repeated the fits with an extinction law with a steeper rise in the UV, using $R_{\mathrm{V}}=2$ instead of $R_{\mathrm{V}}=3.1$ in the parametric description of Cardelli et al. (1989). As expected, $R_{\mathrm{V}}=2$ reduced $\Delta A_{\mathrm{V}}$ on average for giants between 4000 and $5000 \mathrm{~K}^{3}$, but without eliminating the positive average. The other region of the HR diagram where our sample has high extinctions corresponds to luminous warm stars. Here, switching to the steeper $R_{\mathrm{V}}$ tends to increase the discrepancies $\Delta A_{\mathrm{V}}$ between UVB and VIS estimates.

\subsubsection{Systematic differences between best-SED parameters and those based on ULySS and MILES or ELODIE}

As discussed above, the models that reproduce the SEDs of the XSL-spectra best are not always those with the parameters derived by comparison of the absorption line spectra with the empirical libraries MILES or ELODIE. In a small number of cases, this can be traced back to an outlier-type failure of the analysis of the optical spectra, but this is not our main point here. On the contrary, we focus on generic trends, that systematically affect stars of a given part of parameter space and that withstand errors on individual parameters.

Gravity and temperature. A synthetic view of the differences between the parameters $T_{\text {eff }}$ and $\log (g)$ of Paper II and those obtained from the comparison with (reddened) theoretical spectra (at $R=3000$ ), is presented in the HR-diagrams of Fig. 8. Compared to the positions assigned in Paper II in $T_{\text {eff }}$ and $\log (g)$ (black dots), the parameters assigned via the joint comparison of the three arms to GSL-spectra are systematically more dispersed (colored points). More dispersion is expected

\footnotetext{
3 Not below $4000 \mathrm{~K}$, where the fits are poor and dispersion washes out any trend.
} 

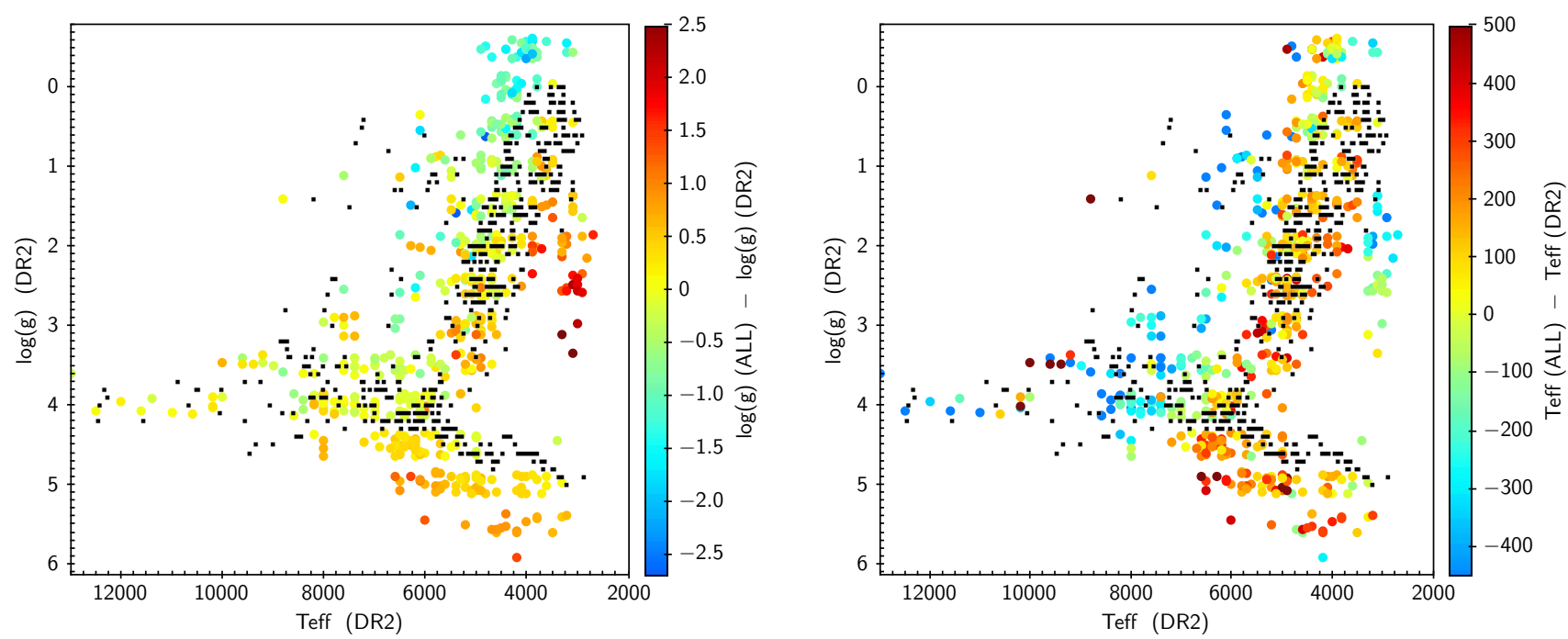

Fig. 8. Differences between input and output gravities and temperatures across the HR diagram. The small black dots locate the XSL data according to our initial parameters, derived from the optical absorption line spectra using ULySS+MILES (Arentsen et al. 2019). The larger dots locate the same XSL data using the best-fit parameters obtained from the global comparison of the empirical spectra (UVB+VIS+NIR) with GSL models, reddened as necessary, at $R=3000$. In the left panel color codes the differences in $\log (g)$ between the two estimates; in the right panel it codes the differences in $T_{\text {eff }}$.

as a result of flux calibration errors in the XSL data combined with degeneracies between parameters, and is exacerbated at low temperatures by the absence of good matches in the model collection (due to the unique adopted extinction law, to the constant abundance ratios in the models, and to numerous other potential discrepancies between the physics implemented in the models and reality). Despite the dispersion, the color-coding in the figure shows systematic trends that are highly significant.

The left panel of Fig. 8 highlights differences between the surface gravities derived from the fits to GSL models and those of the initial guesses. They are responsible for most of the broadening of the main sequence and of the giant branch seen in the GSL-based HR-diagram. For main sequence stars below $\sim 6000 \mathrm{~K}$, the gravities preferred by the comparison to GSL models are larger than the initial guesses (the largest of these values exceed expectations from stellar evolution models). For the red giants between 4000 and $5000 \mathrm{~K}$, the GSL-based gravities are on average lower and this trend is carried mostly by the metal-poor giants $([\mathrm{Fe} / \mathrm{H}] \leqslant-0.5)$. On the other hand, between 5000 and $5500 \mathrm{~K}$, the gap seen in the initial parameters between the main sequence and the giant branch is filled, when using GSL-based parameters, with objects from the lower-luminosity giant branch that are assigned higher gravities. The stars for which GSLbased effective temperatures and gravities are around $3000 \mathrm{~K}$ and $\log (g)=2$ to 3 , are LPVs, for which inspection of the empirical and theoretical spectra shows the models are obviously inadequate (Lançon et al. 2019). We do not discuss these objects further in this paper.

The main trends seen in the comparison between our initial effective temperatures and those from the global GSL-fit, in the right panel of Fig. 8, display a pattern as a function of position that differs from the one seen for the surface gravities. For warm stars along the main sequence or in the transition region between the main sequence and the giant branch (blue loop, blue horizontal branch), the $T_{\text {eff }}$ from GSL-fits is systematically lower than obtained from optical line studies, while differences in the opposite direction are found mostly along the giant branch and at intermediate temperatures on the main sequence (4000 to $7000 \mathrm{~K})$. A comparison between the two panels shows that there is no systematic correlation or anticorrelation between the effects on $\log (g)$ and $T_{\text {eff }}$ that would be valid across the whole HR diagram. Locally, correlations can be found, for instance, at intermediate temperatures on the main sequence, higher gravities are compensated with higher temperatures.

Because the direction of the systematic offsets depend on the area of the HR diagram, we can be confident that they reflect a real difference between observed stars and the models, rather than errors in the data (which should not correlate with position in the HR diagram). The patches over which a coherent trend is observed do not have shapes that would suggest an effect of the sampling of parameter space in the model grid (the general aspect is identical whether or not interpolation is implemented within the grid).

We now repeat the previous comparison, but using best-fits carried out in individual arms (Fig. 9). To ease comparisons between panels and with figures in previous sections, the location of the points in the diagram is now again based on the parameters of Paper II (not on the comparison with GSL); the bottom panels repeat the data from Fig. 8, in this preferred format.

A striking result from this exercise is the similarity between the trends found, whether the fits are based on the UVB, the VIS, the NIR or all three spectral ranges. It shows that certain major systematics are very robust.

While the trends are similar for the different fitting methods (usage of $D$ or of the weighted $\chi^{2}$, areas in the UVB arm excluded or not by a mask, resolution adopted for the fits), the individual estimated parameters depend on these. The errors also depend on the area of the HR diagram considered, with larger errors where the general quality of the fit is poorer, but we provide only a global summary in Table 3 . The methodinduced changes $\delta T_{\text {eff }}, \delta \log (g), \delta[\mathrm{Fe} / \mathrm{H}]$, and $\delta \mathrm{A}_{V}$ are all positively correlated with each other, within a given comparisonexperiment. None of the method-changes erases the trend seen in $\mathrm{A}_{V}(\mathrm{UVB})-\mathrm{A}_{V}(\mathrm{VIS})$ versus $T_{\text {eff }}$.

Metallicity. The differences between the best-fit metallicities found here and the DR2 values from Paper II are displayed 

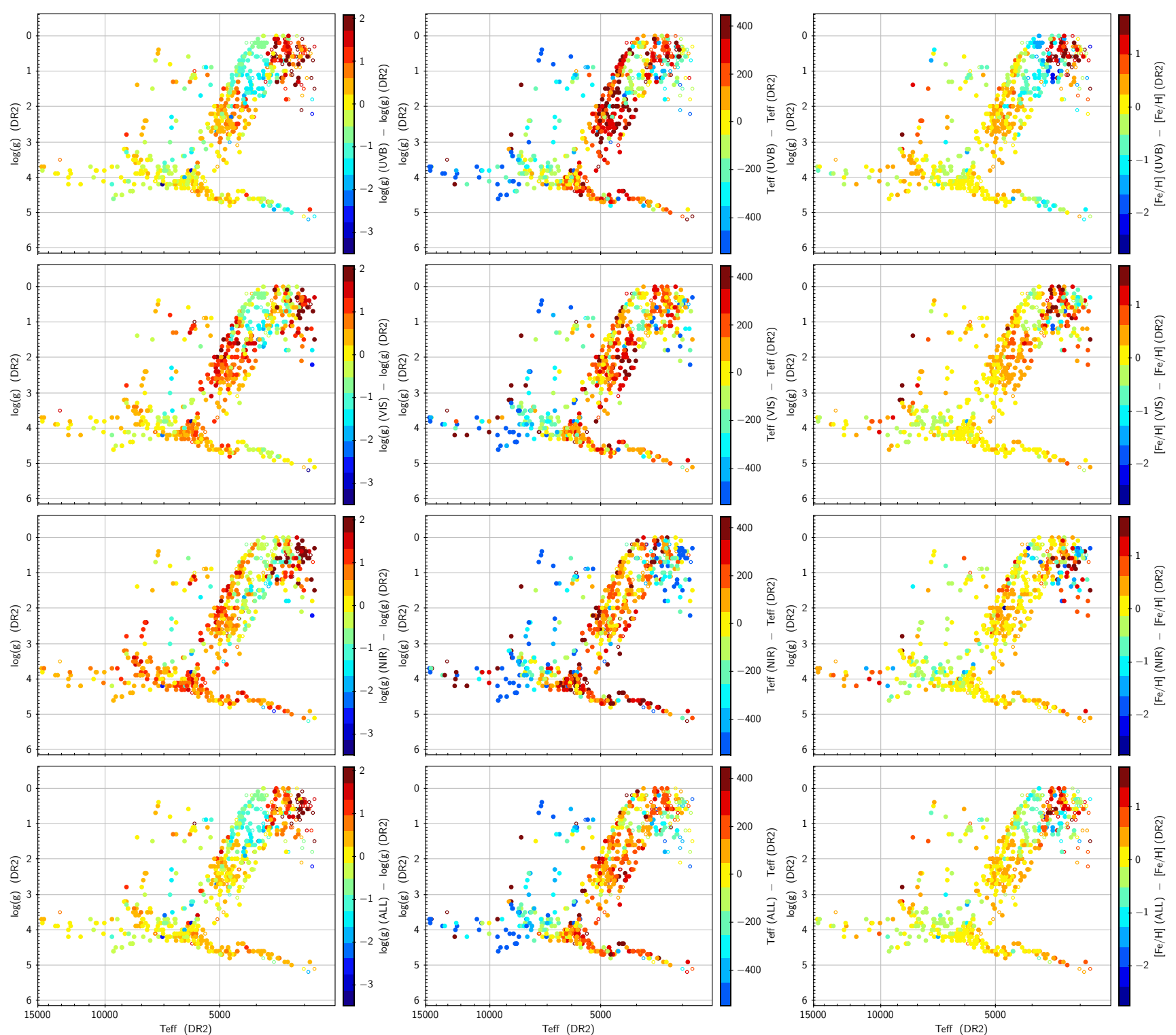

Fig. 9. Trends in the differences between the parameters of DR2 and those from best-matching GSL SEDs as a function of position in the HR diagram. The data in the left column are colored by difference in $\log (g)$, the middle column by difference in $T_{\text {eff }}$, and the right column by difference in $[\mathrm{Fe} / \mathrm{H}]$. From top to bottom, the spectral ranges used for the comparison with GSL are the UVB, the VIS, the NIR, and all three arms of X-shooter.

Table 3. Effects of the fitting method on estimated parameters.

\begin{tabular}{lcccccc}
\hline \hline \multicolumn{2}{c}{$R=500$ vs. $R=3000$} & $\begin{array}{c}R=500, \text { original model grid } \\
\text { vs. interpolated grid }\end{array}$ & $\begin{array}{c}\text { Weighted } \chi^{2} \text { vs. } D, \text { at } R=500 \\
\text { (with slightly different masks) }\end{array}$ \\
\hline Quantity & Mean & Standard deviation & Mean & Standard deviation & Mean & Standard deviation \\
\hline$\delta T_{\text {eff }}(\mathrm{K})$ & 22 & 64 & 0.7 & 123 & 72 & 239 \\
$\delta \log (g)\left(\mathrm{cm} \mathrm{s}^{-2}\right)$ & 0.04 & 0.22 & -0.03 & 0.5 & 0.02 & 0.9 \\
$\delta[\mathrm{Fe} / \mathrm{H}](\mathrm{dex})$ & 0.007 & 0.15 & 0.015 & 0.34 & 0.07 & 0.65 \\
$\delta A_{\mathrm{V}}(\mathrm{mag})$ & 0.001 & 0.08 & 0.03 & 0.34 & 0.16 & 0.49 \\
\hline
\end{tabular}

Notes. Each subsection of the table lists mean parameter differences and standard deviations obtained when switching between the two methods indicated above the column titles. The listed values are based on fits to data from ALL wavelengths of the XSL spectra (UVB + VIS + NIR).

in the right-hand panels of Fig. 9. Over vast areas of the HR diagram, the average local offsets are smaller than 0.5 dex. In particular, main sequence metallicities between 5000 and $7000 \mathrm{~K}$ are similar to those in DR2, despite the systematic offsets in temperature and $\log (g)$ shown in the left-hand panels.
Along the giant branch, the offsets in $[\mathrm{Fe} / \mathrm{H}]$ evolve from typically positive values at high gravity (low luminosity) to typically negative values at low gravity (high luminosity). The higher metallicities in the low luminosity regime contribute to the excessive strength of the metal lines between 490 and $550 \mathrm{~nm}$ 
(UVB arm) that were highlighted as a systematic feature of the spectral residuals in Sect. 5.2.1. It is noteworthy that these higher metallicities did not suffice to solve the apparent issue of lacking absorption at the blue end of the UVB arm, in this regime. At higher luminosities, lower metallicities are selected with our SED-fits; the metal-lines at the red end of the UVB wavelength range tend to match those of the observed spectra better, but on average the synthetic lines remain deeper than those observed even there.

In view of the complex patterns in Fig. 9, an exhaustive analysis would require separate in-depth studies in each subarea of the HR diagram, with tunable models. This is an enormous task, that we postpone until the global study presented here has been repeated with other model collections of the literature.

\subsection{Varying the $[\alpha / \mathrm{Fe}]$ ratio}

In the above, we have not insisted on the $[\alpha / \mathrm{Fe}]$ ratio. When searching for the best-fit with the methods of this paper, the uncertainties on individual derived parameters are too large to allow a robust estimate of $[\alpha / \mathrm{Fe}]$ per star. In particular, the preferred $[\alpha / \mathrm{Fe}]$ ratio depends on the wavelength range used (left panels of Fig. F.1). The UVB range is in principle sensitive to $[\alpha / \mathrm{Fe}]$, via $\mathrm{CaII}, \mathrm{CN}$, and $\mathrm{CH}$ features that are obvious in the residuals. In practice, the UVB range favors GSL models with a super-solar $[\alpha / \mathrm{Fe}]$ almost everywhere in the HR diagram. The VIS range is sensitive to $[\alpha / \mathrm{Fe}]$ in certain regions of parameter space; for instance the $\mathrm{CN}$ bands that are prominent in giants around $4500 \mathrm{~K}$ react to changes in oxygen-proportions via the chemical networks involving C,N, and O (see Lançon et al. 2007; Aringer et al. 2016, for examples of the complex behavior of the $\mathrm{CN}$ bands). The near-infrared calcium triplet $(\sim 880 \mathrm{~nm})$ is also located in the VIS range of X-shooter. The VIS-fits point to super-solar $[\alpha / \mathrm{Fe}]$ mostly for low-metallicity giants and for intermediate temperature metal-poor main sequence stars, but also for about half the stars above $[\mathrm{Fe} / \mathrm{H}]=-0.5$ (Table F.1). The NIR arm, or the SED as a whole, either show no preference or weakly favor solar models in some parts of the HR diagram. Hence, the expected anticorrelation between $[\alpha / \mathrm{Fe}]$ and $[\mathrm{Fe} / \mathrm{H}]$ characteristic of the Milky Way is recovered only when applying a relatively fine-tuned selection to define the "preferred" $[\alpha / \mathrm{Fe}]$.

In the above sections, we have adopted solar-scaled models by default, except for stars for which $\alpha$-enhanced ones were favored in the UVB and not significantly disfavored in the VIS and NIR. The $\alpha$-enhanced subset with this definition contains less than $15 \%$ of the metal-rich stars $([\mathrm{Fe} / \mathrm{H}]>-0.5$ in Arentsen et al. 2019), and about $75 \%$ of the metal-poor stars $([\mathrm{Fe} / \mathrm{H}]<$ -1.5). We refer to Appendix F for details. An attempt to extract $[\alpha / \mathrm{Fe}]$ estimates for the XSL stars from the literature rather than perform our own evaluation was abandoned because of the heterogeneity of the results found in publications. The uncertainty on $[\alpha / \mathrm{Fe}]$ is one of the reasons why we have restricted our discussion to major global trends.

\section{Discussion}

\subsection{Comparison with trends exposed in the literature}

One of the earliest studies comparable to the one we exposed was published by Bertone et al. (2004), who compared ATLAS and PHOENIX models of that time with each other and with more than 300 empirical low-resolution spectra that extended from 350 to $1050 \mathrm{~nm}$. Their method was similar to ours, although they chose to compare logarithms of fluxes, and to correct for Galactic extinction a priori. They concluded that the fit quality was good for spectral type $F$ and earlier and degraded drastically with later spectral types. Today we would soften this judgement to include at least the early $G$ type stars in the ensemble of stars that can be represented with average spectral residuals of a few percent. This was also the conclusion of Coelho (2014) based on ATLAS and MARCS model atmospheres and ATLAS9/SYNTHE synthetic fluxes. In the remainder of this section, we focus on temperatures below this threshold, with an emphasis on giants between 4000 and $5000 \mathrm{~K}$.

It is well known that cool stellar spectra are difficult to model and analyze (Bessell et al. 1989; Westera et al. 2002; Martins \& Coelho 2007; Lebzelter et al. 2012; Short et al. 2012; Davies et al. 2013; Coelho 2014; Franchini et al. 2018). As we cannot possibly review the vast body of pre-existing work exhaustively, we concentrate on comparing the directions of the trends found here with those found previously.

Bertone et al. (2004) compared the temperatures they derived from the low resolution spectra to empirical temperature calibrations as a function of spectral type (based in particular on angular diameter measurements and surface brightnesses). For $\mathrm{K}$ and $\mathrm{M}$ giants, their fit- $T_{\text {eff }}$ were warmer than those of the empirical calibrations by $4-8 \%$, for both giant and dwarf stars. This trend seems to have withstood the passing of time, though its amplitude may be decreasing. Our Fig. 8 shows that the temperatures obtained with more recent PHOENIX models, after a significant update in line opacities in 2008, still lead to higher $T_{\text {eff-values than those of the literature for similar stars (as traced }}$ by the parameters of Paper II). We find that this remains true for any of the UVB, VIS, NIR and ALL fitting-ranges. Our study shows that the discrepancy cannot be blamed on a detail, but has a generic cause that will be fundamental to elucidate.

Similar offsets have been found in other recent studies. For instance, Husser et al. (2016) and Jain et al. (2020) analyzed low-resolution optical spectra of hundreds of stars in the globular cluster NGC $6397([\mathrm{Fe} / \mathrm{H}] \simeq-2)$, by comparing them on one hand with PHOENIX spectra (the GSL collection also used here), on the other with the empirical collections MILES and ELODIE (as was done for XSL in Paper II). Compared to our study, two differences in methodology are noteworthy: stellar gravities were derived prior to the spectroscopic study, from the comparison of the broad band photometry with a theoretical isochrone; and a multiplicative polynomial was allowed to absorb extinction and flux calibration errors. Despite these differences, the trends resemble those obtained here with XSL: the temperatures assigned to giant stars based on GSL are higher by 150 to $200 \mathrm{~K}$ than those obtained with the empirical libraries. The studies in NGC 6397 also found that the $[\mathrm{Fe} / \mathrm{H}]$ assigned with GSL were higher, as expected from the classical temperature-metallicity degeneracy. In our study, this correlation is recovered when fitting only the VIS arm of X-shooter, but it is lost in dispersion when the other wavelength ranges are used.

A comprehensive re-analysis of giant stars in 16 Milky Way clusters, with a range of metallicities, was carried out by Mucciarelli \& Bonifacio (2020). They compared stellar parameters from high resolution optical lines on one hand (excitation and ionization balance, for spectral lines between 480 and $680 \mathrm{~nm}$ ), with parameters from dereddened broad-band colors on the other (metallicity-dependent color- $T_{\text {eff }}$ relations based on the infrared flux method). They found increasing discrepancies between spectroscopic and photometric parameters, with decreasing cluster metallicity. This larger tension at low metallicity between the SEDs and the spectral features is a trend we have also noted in the comparison between XSL and GSL. 
It is difficult to compare the trends between the two studies more quantitatively, because none of the methods used by Mucciarelli \& Bonifacio (2020) corresponds to those used here precisely. The strongest of these trends, seen at low metallicity $([\mathrm{Fe} / \mathrm{H}]<-1.7$ in their sample), is a shift to lower temperatures and lower gravities when switching from photometric to spectroscopic parameters. The signs of these offsets correspond to our results with XSL (though with some dependence on the X-shooter arm and on luminosity in the case of gravity), if we assimilate the parameters of Paper II with Mucciarelli \& Bonifacio's "spectroscopic parameters" and those from the fits to the GSL-SEDs to the "photometric" ones (Figs. 8 and 9). This pairing of analysis methods is certainly not perfect, but it is more reasonable than the alternative pairing would be.

The studies above focus on individual stars or on stellar populations that can be resolved into stars. The two recent articles we now discuss compare empirical and theoretical stellar spectra in the context of the calculation of the integrated spectra of unresolved stellar populations.

Maraston et al. (2020) exploit the empirical library MaS$\operatorname{tar}$ (Yan et al. 2019, $\lambda \lambda$ 362-1035 nm, $R \simeq 1800$ ), and discuss parameters from two estimation methods. One is very similar to the method adopted in Paper II and rests on the MILES library (Chen et al. 2020). The other is based on a comparison with theoretical spectra taken mostly from the BOSZ-ATLAS9 calculations of Mészáros et al. (2012) and Bohlin et al. (2017). For simplicity, we refer to estimates from the latter method as theoretical parameters, as in the original article. Both methods discard information present in the stellar continua, by allowing for a multiplicative polynomial in the fits.

A number of trends found by Maraston et al. are similar to the ones we find here with XSL and GSL, despite different fitting methods and reference models. For instance, the theoretical surface gravities are lower than those based on MILES for luminous red giants, while they tend to be higher for main sequence stars. As in our case, the dispersion of the data points in $\log (g)$ $T_{\text {eff }}$ planes is much larger when using theoretical parameters than when using parameters based on MILES (Figs. 3, 7, 11 of Maraston et al. 2020).

Coelho et al. (2020) confront the dereddened empirical spectra of the MILES library $(\lambda \lambda 354-741 \mathrm{~nm})$ to those they computed for atmosphere models constructed with ATLAS or MARCS, assuming spherical symmetry for giants below $4000 \mathrm{~K}$. They adopt stellar parameters from the literature, from references that mostly match those adopted in Paper II. Hence their results can be compared to our discussion in Sect. 5.1, albeit with a difference in the extinction correction. Their goodness-of-fit results are similar to those we obtain here: the fit-quality starts to degrade below $5000 \mathrm{~K}$. Interestingly, the two fit-discrepancy measures they define reach their minimum at different effective temperatures. One is a weighted $\chi^{2}$ that exploits the empirical noise spectrum, the other a summed fractional difference $\tilde{\Delta}$ similar in essence to our $D$. Like our $D$, their $\tilde{\Delta}$ rises quickly below $5000 \mathrm{~K}$ while the $\chi^{2}$ only picks up large values below about $4000 \mathrm{~K}$. This confirms our impression that $D$ or $\tilde{\Delta}$ might be too sensitive to regions of low flux in the blue part of the spectrum of cool stars.

For more detailed comparisons, Coelho et al. (2020) refer to Coelho (2014). The spectral residuals shown there for temperatures below $5000 \mathrm{~K}$ are of amplitudes that seem roughly comparable to those found here between XSL and GSL. Coelho (2014) experiment in using MILES to re-evaluate parameters starting from their synthetic optical spectra. The results however are presented (and were archived) in a very compact way that does not allow us to directly compare trends with those found here (their Fig. 12). We intend, instead, to compare XSL and the models of Coelho (2014) directly in the future.

\subsection{Limitations of the models}

The spectral properties of cool stellar models depend on numerous parameters and even extensive grids are inevitably limited. The systematic offsets between our best-SED parameters and those of Paper II are likely due to a combination of issues in the model ingredients and systematics in the parameters assigned to MILES and ELODIE spectra in the literature. The complex discrepancies between empirical and best-fit theoretical spectra, in particular below $5000 \mathrm{~K}$, demonstrate that the models carry their share of responsibility, and Sect. 6.1 shows that this is not an isolated problem of the GSL collection. Here, we examine a few of the likely issues that affect GSL in particular, and a number of other collections by generalization.

In spherical symmetry, the assumed relation between mass and position in the HR-diagram sets the extension of the atmosphere models, which affects molecular band depths and colors (Fig. 8 of Aringer et al. 2016; Hauschildt et al. 1999; Gustafsson et al. 2008). This is important only at low gravities. In GSL, the masses assumed at low temperatures do not exceed a few solar masses. This leads to relatively large atmospheric extensions, to relatively low luminosities, and it is more adequate for giants than for red supergiants. Models more specifically designed for red supergiants, with masses of $15 M_{\odot}$ were confronted with observations across the optical and near-infrared spectrum by Lançon et al. (2007) and Davies et al. (2013). The former are based on PHOENIX models and adopt surface abundances specifically taylored for red supergiants but with line lists that are obsolete compared to those used here; the latter are based on MARCS models with solar scaled abundances and prior assumptions on metallicity and gravity. Apart from the general conclusion that the features and energy distributions are difficult to match simultaneously for cool red supergiants, it is difficult to compare the two studies. In particular, the "optical" wavelength range used by Davies et al. for their comparisons differs from our VIS or UVB range: the range they select is specifically sensitive to $\mathrm{TiO}$ absorption and excludes the red end of our VIS range where $\mathrm{CN}$ bands can be dominant (around $4500 \mathrm{~K}$ ). The two molecules form bands at different depths in the atmosphere ( $\mathrm{TiO}$ further out), and this makes the comparison sensitive to the (different) extensions of the atmospheres in the two collections. Future comparisons should exploit independent measurements of luminosity and include models with a range of masses in the low-gravity regime.

The assumption of Local Thermal Equilibrium (in GSL and in many synthetic spectral libraries) impacts the stratification of atmospheres and subsequently the line strengths, line profiles, and SEDs. According to Short \& Hauschildt (2003) and Short et al. (2012), the tendency between 4000 and $5000 \mathrm{~K}$ is for nonLTE spherical models to have slightly higher gas temperatures in their atmospheres (i.e., at optical depths smaller than one),

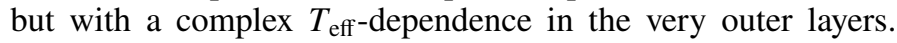
This makes it difficult to identify "rule-of-thumb" behaviors for molecular bands. The studies also suggest that non-LTE models produce more short-wavelength flux than LTE models, which we anticipate would increase the SED-issues that we have illustrated in this paper via the discrepancies between extinction estimates in the UVB and in the VIS range of the spectra.

Although atmosphere models generally include turbulent energy transport via the mixing-length theory, the effects of 
turbulence are by no means treated completely in current calculations. Some of the additional velocity dispersion is accounted for in the spectral synthesis via a micorturbulence parameter. Its values affect the importance of line blanketing, as well as relative strengths of saturated and unsaturated lines in spectra (Tsuji 1976; Short \& Hauschildt 2010; Lançon \& Hauschildt 2010). But the surfaces of red giants and supergiants have convective patterns on larger scales that translate into distributions of surface temperatures and effective gravities, and their dynamical behavior may lead to more extended atmospheres, outer molecular envelopes, or the formation of dust. Further developments of 3D-calculations (e.g., Chiavassa et al. 2018; Höfner \& Freytag 2019) will probably be necessary to assess the relevance of these processes to the relation between SEDs and spectral features. In particular it is unclear whether significant systematic effects could be produced above $T_{\text {eff }} \simeq 4000 \mathrm{~K}$.

We now move from physical to chemical considerations. In the GSL library, the metal abundance ratios are fixed except for $[\alpha / \mathrm{Fe}]$. In this article, we have explored only two values of this ratio, but based on this exploration we do not expect $[\alpha / \mathrm{Fe}]$ by itself to resolve the tensions between data and models that we have discussed. It seems necessary to also account for dredgeup, or more generally to use surface abundances that account for stellar evolution. Dredge-up in particular affects C, N, O abundances (e.g., Georgy et al. 2013a), and these provide some of the dominant molecular features between 4000 and $5000 \mathrm{~K}$ (Lançon et al. 2007; Aringer et al. 2016). The clear ${ }^{13} \mathrm{CO}$ bandheads seen beyond $2.3 \mu \mathrm{m}$ in many XSL spectra of giants also signal dredgeup and are not reproduced by the models. At low metallicity, carbon-enhancements are common in the Milky Way; this may contribute to explain the tension we observed between spectral features and SEDs in this regime, and it should be accounted for in future synthetic spectral libraries.

Another common particularity of synthetic spectral libraries, including GSL, is a constant value of the $\mathrm{He} / \mathrm{H}$ ratio at all metallicities. This is at odds with the positive $\Delta Y / \Delta Z$ representative of the global chemical evolution of a galaxy, that is usually assumed in stellar evolution calculations (Bressan et al. 2012; Georgy et al. 2013b). The helium fraction impacts the stellar structure more than it affects the calculated spectrum for a given stellar structure, hence the latter is often neglected (Girardi et al. 2007; Dotter et al. 2008; Chantereau et al. 2016; Chung et al. 2017). Indeed, at temperatures above about $4500 \mathrm{~K}$ and in all but the bluest photometric passbands, moderate He-enhancements $(\Delta Y \simeq+0.1$ at a given $[\mathrm{M} / \mathrm{H}])$ change bolometric corrections by less than $1 \%$ in plane-parallel models (Girardi et al. 2007). However changes induced by such He-enhancements on the bolometric corrections and colors can be larger than 5\% below $4000 \mathrm{~K}$, and it seems that systematic studies with spherical models remain to be carried out.

Finally, we note that even with the correct abundances mismatches at some level are expected from incomplete opacity data. The PHOENIX calculations include lists of empirically validated spectral lines but also theoretical lists, in which line wavelengths and strengths are given with poor precision. These so-called "predicted lines" are mostly weak ones, but are very numerous. Once the spectra, initially calculated with a spectral sampling resolution near 500000 , are smoothed to $R=3000$, they manifest to first order as a pseudo-continuous absorption. As shown by Coelho (2014), albeit with a different spectral synthesis code than used here and not with exactly the same opacity input, this effect increases towards blue wavelengths and towards low effective temperatures. Considering the uncertainties associated with the predicted lines, it is very likely that shortcomings in current lists contribute to the tension that, in our study, led to the apparent need for more reddening in the UVB arm of Xshooter than in the VIS arm. In Sect. 5.2.1 and Fig. 5 we noticed that between 4000 and $5000 \mathrm{~K}$, and at wavelengths shorter than about $430 \mathrm{~nm}$, the best-fit models for dwarfs or low-luminosity giants seemed to have excess flux in between the strongest lines; this might argue in favor of missing pseudo-continuous opacity (although considering the degeneracies in the fitting exercise, other explanations are also possible). The effect is not as clear at higher metallicities (Fig. 6), where uncertainties in the very numerous molecular lines, combined with degeneracies in the model parameter selection, may mask it.

\subsection{Anticipated effects on the integrated colors of simulated SSPS}

In this section, we briefly discuss how errors on fundamental parameters, of amplitudes comparable to the differences between the parameters of Paper II and those we obtained from matching the global SEDs with GSL models, may affect the contributions of various types of stars to the integrated light of a stellar population. Population synthesis models based on XSL spectra are in preparation (Verro et al.). Here, we approach the question by comparing the bolometric corrections of the synthetic spectra that correspond respectively to the DR2-parameters and to the SED-fit parameters (fits to all wavelengths). This avoids any calculation of bolometric corrections from the XSL-spectra themselves, which despite their broad spectral range do not cover the full spectrum of photospheric emission. We define the bolometric correction for photometric passband $X$ as $B C_{X}=M_{\mathrm{bol}}-$ $M_{X}$.

The synthetic spectra distributed in GSL are wavelengthdependent surface fluxes, integrated over all directions. After integration over the photometric passband of interest, the fluxes must be multiplied by the area of the stellar surface to allow a comparison with the total luminosity of the model. For stars with extended atmospheres, the relevant outer radius is larger than the effective radius (which relates luminosity to effective temperature via the Stefan Boltzmann law), but only the latter is present in the default headers of the data files. The correct outer radii were extracted from intermediate data files of the PHOENIX archives ${ }^{4}$ and bolometric corrections computed. The procedure does not include extinction, because most population synthesis codes use extinction-free libraries of spectra or colors; spectra such as those of XSL would be de-reddened before implementation in a population synthesis code.

As illustrated in Fig. 10, the effects of changes in fundamental parameters of the amplitude discussed in this paper are of variable importance depending on the passband selected and on the region in the HR diagram. In the $U$ and $K$ bands (top left and bottom right panels), a simple dichotomy appears between main sequence stars below $6500 \mathrm{~K}$ and giants on one hand, and warmer stars on the other. This essentially mirrors the distribution of $T_{\text {eff }}$ (ALL)- $T_{\text {eff }}$ (DR2) across the HR diagram (Fig. 9). In the analysis of the integrated light of a galaxy, the dichotomy would directly affect the proportions of young and old stellar subpopulations.

The bolometric corrections vary less across the HR diagram in the optical passbands $V$ and $I$. The dichotomy seen in $U$ and $K$ is weak or absent, and replaced with more subtle local effects. For instance, a gradient in $\triangle \mathrm{BC}_{X} \equiv \mathrm{BC}_{X}(\mathrm{ALL})-\mathrm{BC}_{X}(\mathrm{DR} 2)$ is

\footnotetext{
4 Unfortunately a few of the intermediate files are lost, so the parameter space covered here is slightly more restricted than in previous sections.
} 

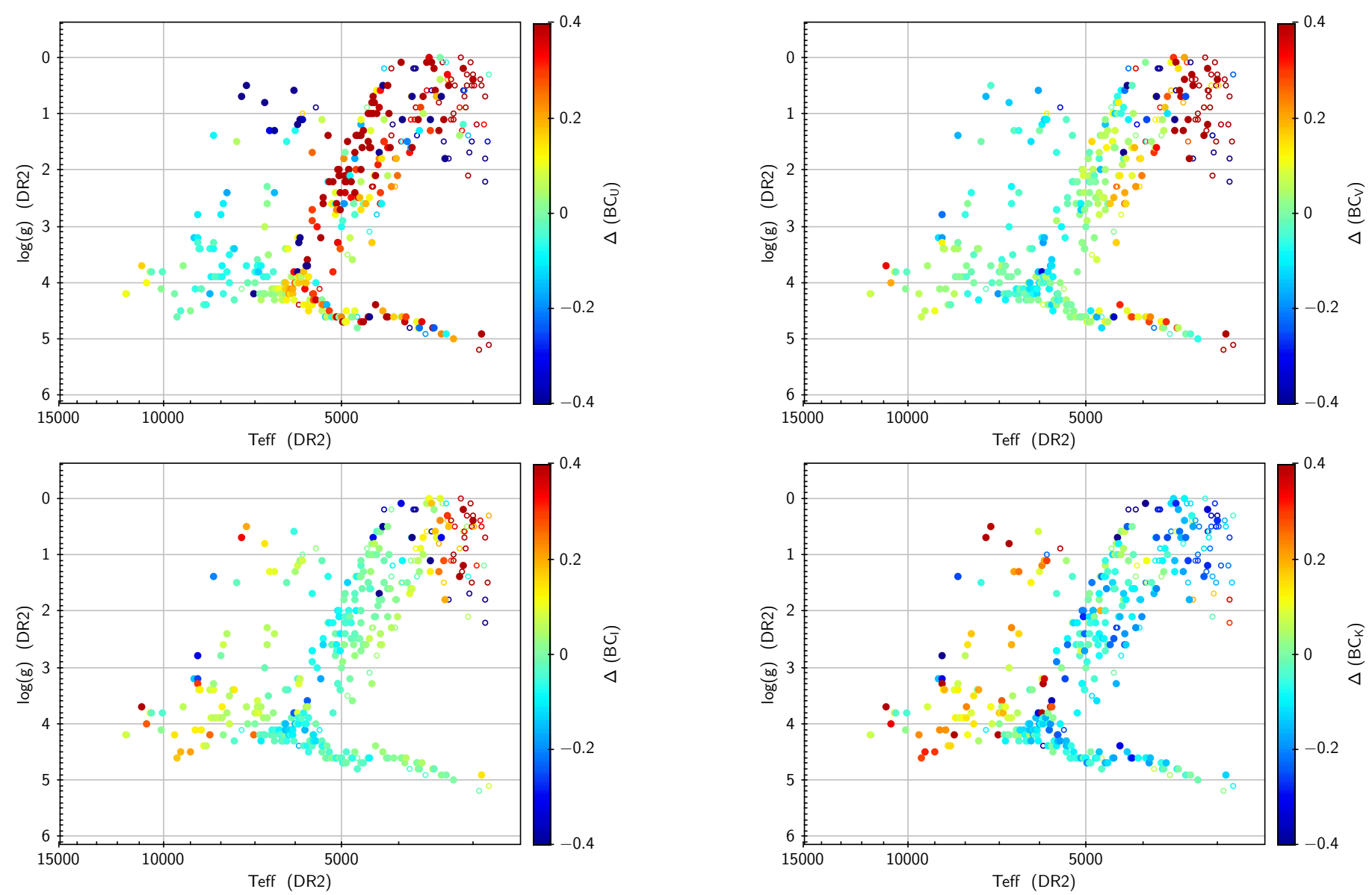

Fig. 10. Effects of parameter-differences between Arentsen et al. (2019, DR2) and SED-fits with GSL spectra (ALL wavelengths) on bolometric corrections: $\triangle \mathrm{BC}_{X}=\mathrm{BC}_{X}(\mathrm{ALL})-\mathrm{BC}_{X}(\mathrm{DR} 2)$. The four panels are for the bolometric corrections to classical $U, V, I$, and $K$ bands, respectively. The layout of the figure is as in Fig. 1.

seen across the red giant branch in the $V$ band, and with lower contrast in the $I$ band (or in the $z$ band; not shown). The temperature of the giant branch depends on metallicity, and metallicity estimates are usually undertaken at optical wavelengths. Hence, the gradient in $\triangle \mathrm{BC}_{V}$ across the giant branch may translate into uncertainties in proportions of metal poor and metal rich stars.

Among the four panels of Fig. 10, the I-band is most relevant to current studies of the stellar Initial Mass Function (IMF) of galaxies based on their integrated light (bottom left panel; the corresponding diagram for the $z$-band, not shown, is very similar). Such studies measure gravity-sensitive features of cool stars to constrain the ratio of dwarfs to giants, and focus on the red to near-infrared wavelengths at which the contribution of cool dwarfs to the total emission of a population is maximal (van Dokkum \& Conroy 2011; La Barbera et al. 2017). The systematic offsets in parameters that we have discussed in this paper would affect such studies if $\triangle \mathrm{BC}_{I}$ differed between giants and dwarfs. No strong difference of that type is seen, but only complete population synthesis models could quantify the small effects that may still be present and that may be relevant considering the high accuracies required in IMF-studies.

\section{Conclusions}

We have compared the spectra of XSL-DR2 (Gonneau et al. 2020) with the theoretical spectra of the Göttingen Spectral Library (Husser et al. 2013), at low and intermediate resolution $(R=500$ and 3000$)$, for wavelengths extending from the near ultraviolet to the near-infrared $(\sim 350 \mathrm{~nm}$ to $2.45 \mu \mathrm{m})$, with the main aim of determining whether a good match to both the energy distributions and the spectral features can be obtained simultaneously.

(i) When adopting the stellar parameters derived by Arentsen et al. (2019), the low resolution SEDs of XSL spectra and those of (reddened) GSL PHOENIX-spectra are comparable over a wide part of the HR diagram, and the comparison of spectral features at $R=3000$ is very good overall at warm effective temperatures. However, systematic discrepancies between the empirical and theoretical energy distributions appear below $5000 \mathrm{~K}$. Between 4000 and $5000 \mathrm{~K}$, when extinction is optimized for a best match in the VIS range of X-shooter $(0.55-1 \mu \mathrm{m})$, the empirical spectra in the UVB range $(<550 \mathrm{~nm})$ tend to be redder than the models and the empirical spectra in the NIR range tend to be bluer than the models. The effect is more obvious at low metallicity than at near-solar metallicity. We recall that Arentsen et al. (2019) compared the optical part of the XSL absorption line spectra, at $R \simeq 2000$, with existing empirical libraries (ELODIE/MILES), for which published parameters are the result of a compilation of varied detailed studies in the literature. Those libraries are a frequent reference in stellar population studies (Le Borgne et al. 2004; Falcón-Barroso et al. 2011; Coelho et al. 2020; Chen et al. 2020).

(ii) When the GSL library is searched for the best-fitting reddened model for each XSL data-set, good matches can be found down to about $4000 \mathrm{~K}$. By "good matches", we mean that the residuals (XSL-GSL) at $R=500$ are on average flat across 
the whole spectral range of XSL. However, spectral features, in particular in the UVB arm, are not always reproduced well when the SED is. This is in particular true between 4000 and $5000 \mathrm{~K}$, where it remains difficult to reproduce the SED and spectral features simultaneously. Below $4000 \mathrm{~K}$, only a handful of empirical spectra find roughly acceptable matches in the synthetic collection, and we have therefore refrained from discussing trends there. We refer to Lançon et al. (2019) for a preliminary discussion of the coolest, frequently variable giants.

The XSL-GSL discrepancies between 4000 and $5000 \mathrm{~K}$ manifest as differences between the parameters obtained from the three X-shooter arms taken separately and as features in the low or intermediate resolution residuals. Models selected by fitting only one arm are not usually a good representation of the observed spectrum as a whole and this is not explained by flux calibration errors alone. There is a real inconsistency between the theoretical spectral features and SEDs, for which potential causes are multiple (inadequate element abundances, possible departures from LTE, simple assumptions in the spherical models for properties such as microturbulence, atmospheric extension or stellar mass as a function of position in the HR diagram, shortcomings in fundamental data such as line lists, etc.).

We summarize a few of the differences between arms as follows. Between 4000 and $5000 \mathrm{~K}, \Delta A_{\mathrm{V}} \equiv A_{\mathrm{V}}(\mathrm{UVB})-A_{\mathrm{V}}$ (VIS), derived from the SED-fits with the standard extinction law of Cardelli et al. (1989), is in general positive. This difference is reduced, but not erased, when using $R_{\mathrm{V}}=2$ instead of $R_{\mathrm{V}}=3.1$. But we do not expect such an extreme extinction law to apply to many XSL stars (Schlafly et al. 2016) and more generally we do not expect the modeling of galactic extinction to resolve the inconsistencies. $\Delta A_{\mathrm{V}}$ correlates with $T_{\text {eff }}(\mathrm{UVB})-T_{\text {eff }}(\mathrm{VIS})$, but with some dependence on position in the HR diagram; $T_{\text {eff }}(\mathrm{UVB})-T_{\text {eff }}$ (VIS) is largest for the metal-poor giants in the sample. Along much of the main sequence, $\log (g)$ (NIR) is found larger than $\log (g)$ (VIS), while the opposite trend is true on the low-luminosity giant branch.

The systematic features in the spectral residuals (difference XSL-GSL at $R=500$ or $R=3000$ ) are more easily isolated at low metallicity where features are not so numerous. Between 4000 and $5000 \mathrm{~K}$, the models selected based on matches at all Xshooter wavelengths tend to lack opacity in between the strongest absorption features at the blue end of the UVB range $(<400 \mathrm{~nm})$, while their strongest individual metal lines at the red end of the UVB range $(>490 \mathrm{~nm})$ are generally too deep. Also, differences in the depth of molecular bands and $\mathrm{Ca}$ or $\mathrm{Mg}$ lines suggest that the abundances of light elements and $\alpha$-elements may need tuning.

(iii) There are systematic offsets between the parameters of the best-fitting SEDs and those based on the comparison with ELODIE/MILES in Arentsen et al. (2019), even when one accounts for the uncertainties associated with the differences between arms mentioned above. These systematic offsets however depend in a complex way on position in the HR-diagram and they cannot be summarized with a small set of constants. For example, at non-extreme temperatures along the main sequence the best-fitting reddened GSL-SEDs have higher temperatures and gravities than the DR2-values; on the low-luminosity half of the giant branch they have higher temperatures, gravities and metallicities; on the more luminous half of the giant branch they have higher temperatures but lower gravities and metallicities.

Because similar trends with position in the HR diagram appear independently of the spectral arm used (UVB, VIS, NIR, or ALL), it is unlikely that they result from a data artifact, or from modeling issues related to a specific spectral feature, or even a difference in individual abundances. Difference in the parameter scales of the two reference collections seem to play a dominant role.

It is interesting that the trends found here resemble those of other work with empirical and theoretical reference libraries rather well. Maraston et al. (2020) provide a recent example, where the MILES library was used as an empirical reference, and ATLAS and MARCS models for the theoretical one. Jumping to the conclusion that the parameters of ELODIE/MILES should be re-assessed would be premature. Indeed, the parameters from the "theoretical approach" also suffer from a number of caveats that lead to large internal errors, as recalled in (ii) above. We refrain from publishing individual parameters here for this very reason. What our study highlights again, is the need for improvement in the synthetic spectral libraries, which in fact underly both parameter estimate methods in different ways. On one hand, the so-called "empirical" libraries have associated parameters that come from the analysis of ionization and excitation equilibria using isolated lines of metals in high-resolution spectroscopic data, or for cool stars on stellar radii or the infrared flux method. Hence they use only a small part of the full information present in modern empirical spectra. More generic "full spectrum fitting methods", on the other hand, exploit a broader range of data, but that makes them more sensitive to the physical completeness and the internal consistency of the spectral calculations.

(iv) We have explored only models at $[\alpha / \mathrm{Fe}]=0$ and $[\alpha / \mathrm{Fe}]=0.4$. As expected, low metallicity giant star spectra, even at low resolution, on average prefer $\alpha$-enhanced models. Because of degeneracies between the effects of $[\alpha / \mathrm{Fe}],[\mathrm{Fe} / \mathrm{H}], \log (g)$ and $T_{\text {eff }}$, the differences in the quality-of-fit measurements are usually small between the model-fits at the two values of $[\alpha / \mathrm{Fe}]$. Differences between the parameters derived from various arms are in general larger than the differences due to the change of 0.4 in $[\alpha / \mathrm{Fe}]$. Hence our method by itself does not allow us to provide automatic $[\alpha / \mathrm{Fe}]$ estimates for individual stars. This would require a one-by-one adjustment of the chemical abundances, including those of $\mathrm{C}, \mathrm{N}$, and $\mathrm{O}$ which shape the molecules that are responsible for the largest residuals in the data-model comparisons.

(v) Consequences of the highlighted trends are discussed via bolometric corrections. As illustrated recently by Coelho et al. (2020) or Maraston et al. (2020), local dispersion in estimated parameters (random errors near a given position of the HR diagram) have little impact on predicted properties of stellar populations, but systematic effects do (Percival \& Salaris 2009). The systematic offsets between parameters obtained with empirical or theoretical reference libraries translate into differences in bolometric corrections, that directly affect the relative contributions of different stars at different wavelengths. When the differences in bolometric corrections depend on position in the HR-diagram in a systematic way, they will affect the interpretation of galaxy spectra. From the trends observed, we expect uncertainties on $\mathrm{BC}_{U}$ and $\mathrm{BC}_{K}$ (due to method-dependencies of stellar parameters) to affect mainly the estimated proportions of young and old stars, and those on $\mathrm{BC}_{V}$ and $\mathrm{BC}_{I}$ the proportions of stars of different metallicities.

Discrepancies between synthetic and empirical spectra are a limiting factor in our interpretation of the light received from objects at all distances in the Universe and much investment in stellar astrophysics is still needed. Collections such as XSL should be expanded and used to assess new model grids. But in parallel we believe it will be necessary to focus on small samples of reference stars in key locations of the HR diagram, and attempt to optimize models for these objects. For 
instance, an extension of the Gaia FGK Benchmark Star project (Blanco-Cuaresma et al. 2014) to the near-infrared would be most welcome.

Acknowledgements. This work was supported in part by grant ANR-19-CE310022 (POPSYCLE) of the French Agence Nationale de la Recherche, as well as by the Programme National Cosmology et Galaxies (PNCG) of CNRS/INSU with INP and IN2P3, co-funded by CEA and CNES, and by the Programme National de Physique Stellaire (PNPS) of CNRS/INSU. A.V and J. F-B acknowledge support through the RAVET project by the grant AYA2016-77237-C3-1-P from the Spanish Ministry of Science, Innovation and Universities (MCIU) and through the IAC project TRACES which is partially supported through the state budget and the regional budget of the Consejería de Economía, Industria, Comercio y Conocimiento of the Canary Islands Autonomous Community. RFP acknowledges financial support from the European Union's Horizon 2020 research and innovation program under the Marie Skłodowska-Curie grant agreement No. 721463 to the SUNDIAL ITN network. We made extensive usage of the Topcat and Stilts tools (Taylor 2005) and we thank M. Taylor for his attention to questions from users. We also made use of the VizieR catalogue access tool, CDS, Strasbourg, France (DOI: 10.26093/cds/vizier); the original description of the VizieR service was published by Ochsenbein et al. (2000). We thank C. Noûs and the Cogitamus Institute, France (http://www.cogitamus.fr/), and through them the research community as a whole, for their contributions to this work

\section{References}

Arentsen, A., Prugniel, P., Gonneau, A., et al. 2019, A\&A, 627, A138

Aringer, B., Girardi, L., Nowotny, W., Marigo, P., \& Bressan, A. 2016, MNRAS, 457, 3611

Asplund, M., Grevesse, N., Sauval, A. J., \& Scott, P. 2009, ARA\&A, 47, 481

Baldwin, C., McDermid, R. M., Kuntschner, H., Maraston, C., \& Conroy, C. 2018, MNRAS, 473, 4698

Bertone, E., Buzzoni, A., Chávez, M., \& Rodríguez-Merino, L. H. 2004, AJ, 128, 829

Bessell, M. S., Brett, J. M., Scholz, M., \& Wood, P. R. 1989, A\&AS, 77, 1

Blanco-Cuaresma, S., Soubiran, C., Jofré, P., \& Heiter, U. 2014, A\&A, 566, A98

Bohlin, R. C., Mészáros, S., Fleming, S. W., et al. 2017, AJ, 153, 234

Bressan, A., Marigo, P., Girardi, L., et al. 2012, MNRAS, 427, 127

Cardelli, J. A., Clayton, G. C., \& Mathis, J. S. 1989, ApJ, 345, 245

Cenarro, A. J., Peletier, R. F., Sánchez-Blázquez, P., et al. 2007, MNRAS, 374, 664

Chantereau, W., Charbonnel, C., \& Meynet, G. 2016, A\&A, 592, A111

Chen, Y.-P., Trager, S. C., Peletier, R. F., et al. 2014, A\&A, 565, A117

Chen, Y.-P., Yan, R., Maraston, C., et al. 2020, ApJ, 899, 62

Chiavassa, A., Casagrande, L., Collet, R., et al. 2018, A\&A, 611, A11

Chung, C., Yoon, S.-J., \& Lee, Y.-W. 2017, ApJ, 842, 91

Coelho, P. R. T. 2014, MNRAS, 440, 1027

Coelho, P. R. T., Bruzual, G., \& Charlot, S. 2020, MNRAS, 491, 2025

Dahmer-Hahn, L. G., Riffel, R., Rodríguez-Ardila, A., et al. 2018, MNRAS, 476, 4459

Dametto, N. Z., Riffel, R., Colina, L., et al. 2019, MNRAS, 482, 4437

Davies, B., Kudritzki, R.-P., Plez, B., et al. 2013, ApJ, 767, 3

Dotter, A., Chaboyer, B., Jevremović, D., et al. 2008, ApJS, 178, 89

Falcón-Barroso, J., Sánchez-Blázquez, P., Vazdekis, A., et al. 2011, A\&A, 532, A95

Franchini, M., Morossi, C., Di Marcantonio, P., et al. 2018, ApJ, 862, 146

Georgy, C., Ekström, S., Eggenberger, P., et al. 2013a, A\&A, 558, A103
Georgy, C., Ekström, S., Granada, A., et al. 2013b, A\&A, 553, A24

Girardi, L., Castelli, F., Bertelli, G., \& Nasi, E. 2007, A\&A, 468, 657

Gonneau, A., Lyubenova, M., Lançon, A., et al. 2020, A\&A, 634, A133

Gonzalez, O. A., Rejkuba, M., Zoccali, M., et al. 2011, A\&A, 530, A54

Gustafsson, B., Edvardsson, B., Eriksson, K., et al. 2008, A\&A, 486, 951

Hauschildt, P. H., Allard, F., Ferguson, J., Baron, E., \& Alexander, D. R. 1999, ApJ, 525, 871

Heiter, U., \& Eriksson, K. 2006, A\&A, 452, 1039

Höfner, S., \& Freytag, B. 2019, A\&A, 623, A158

Husser, T.-O., Wende-von Berg, S., Dreizler, S., et al. 2013, A\&A, 553, A6

Husser, T.-O., Kamann, S., Dreizler, S., et al. 2016, A\&A, 588, A148

Jain, R., Prugniel, P., Martins, L., \& Lançon, A. 2020, A\&A, 635, A161

Koleva, M., Prugniel, P., Bouchard, A., \& Wu, Y. 2009, A\&A, 501, 1269

La Barbera, F., Vazdekis, A., Ferreras, I., et al. 2017, MNRAS, 464, 3597

Lançon, A., \& Hauschildt, P. H. 2010, in Astronomical Society of the Pacific Conference Series, 425, Hot and Cool: Bridging Gaps in Massive Star Evolution, eds. C. Leitherer, P. D. Bennett, P. W. Morris, \& J. T. Van Loon, 61

Lançon, A., Hauschildt, P. H., Ladjal, D., \& Mouhcine, M. 2007, A\&A, 468, 205 Lançon, A., Gonneau, A., Trager, S. C., et al. 2019, in IAU Symposium, 343, eds. F. Kerschbaum, M. Groenewegen, \& H. Olofsson, 309

Lanz, T., \& Hubeny, I. 2007, ApJS, 169, 83

Le Borgne, D., Rocca-Volmerange, B., Prugniel, P., et al. 2004, A\&A, 425, 881

Lebzelter, T., Heiter, U., Abia, C., et al. 2012, A\&A, 547, A108

Madau, P., \& Dickinson, M. 2014, ARA\&A, 52, 415

Maraston, C., Hill, L., Thomas, D., et al. 2020, MNRAS, 496, 2962

Martins, L. P., \& Coelho, P. 2007, MNRAS, 381, 1329

Mészáros, S., Allende Prieto, C., Edvardsson, B., et al. 2012, AJ, 144, 120

Moutard, T., Arnouts, S., Ilbert, O., et al. 2016, A\&A, 590, A103

Mucciarelli, A., \& Bonifacio, P. 2020, A\&A, 640, A87

Nidever, D. L., Hasselquist, S., Hayes, C. R., et al. 2020, ApJ, 895, 88

Ochsenbein, F., Bauer, P., \& Marcout, J. 2000, A\&AS, 143, 23

Percival, S. M., \& Salaris, M. 2009, ApJ, 703, 1123

Plez, B., Brett, J. M., \& Nordlund, A. 1992, A\&A, 256, 551

Powalka, M., Lançon, A., Puzia, T. H., et al. 2016, ApJS, 227, 12

Powalka, M., Lançon, A., Puzia, T. H., et al. 2017, ApJ, 844, 104

Prugniel, P., \& Soubiran, C. 2001, A\&A, 369, 1048

Prugniel, P., Vauglin, I., \& Koleva, M. 2011, A\&A, 531, A165

Riffel, R., Rodríguez-Ardila, A., Brotherton, M. S., et al. 2019, MNRAS, 486, 3228

Roth, M. M., Sandin, C., Kamann, S., et al. 2018, A\&A, 618, A3

Sánchez-Blázquez, P., Peletier, R. F., Jiménez-Vicente, J., et al. 2006, MNRAS, 371, 703

Schlafly, E. F., Meisner, A. M., Stutz, A. M., et al. 2016, ApJ, 821, 78

Scholz, M. 1985, A\&A, 145, 251

Schönebeck, F., Puzia, T. H., Pasquali, A., et al. 2014, A\&A, 572, A13

Sharma, K., Prugniel, P., \& Singh, H. P. 2016, A\&A, 585, A64

Short, C. I., \& Hauschildt, P. H. 2003, ApJ, 596, 501

Short, C. I., \& Hauschildt, P. H. 2010, ApJ, 718, 1416

Short, C. I., Campbell, E. A., Pickup, H., \& Hauschildt, P. H. 2012, ApJ, 747, 143

Soubiran, C., Le Campion, J.-F., Brouillet, N., \& Chemin, L. 2016, A\&A, 591, A118

Taylor, M. B. 2005, in Astronomical Society of the Pacific Conference Series, 347, Astronomical Data Analysis Software and Systems XIV, eds. P. Shopbell, M. Britton, \& R. Ebert, 29

Taylor, E. N., Hopkins, A. M., Baldry, I. K., et al. 2011, MNRAS, 418, 1587

Tsuji, T. 1976, PASJ, 28, 543

van Dokkum, P. G., \& Conroy, C. 2011, ApJ, 735, L13

Westera, P., Lejeune, T., Buser, R., Cuisinier, F., \& Bruzual, G. 2002, A\&A, 381, 524

Yan, R., Chen, Y., Lazarz, D., et al. 2019, ApJ, 883, 175 
A. Lançon et al.: A comparison between X-shooter spectra and PHOENIX models across the HR-diagram

\section{Appendix A: Example of the diagnostic plots produced for model-data comparisons}

Figures A.1, A.2, and A.3, and A.4 show the match between the models and the data, in the case of HD 111515 (XSL spectrum X0901), plotted at $R=3000$. This is a main sequence star with parameters relatively close to those of the sun. The fits to individual arms and the global fit provide consistent results considering the errors apparent as dispersion among the parameters with low- $\chi^{2}$ values in Fig. A.4; these parameters are slightly offset from to those of Arentsen et al. (2019) (5383 K, $\log (g)=4.4,[\mathrm{Fe} / \mathrm{H}]=-0.6)$. The value of $D($ Eq. $(1))$ with the latter parameters, for ALL wavelengths and at $R=500$, was 0.073 , and it is 0.059 for the best fit. In Sect. 5.1 we describe such fits as consistent with flux-calibration errors at the $2-\sigma$ level.
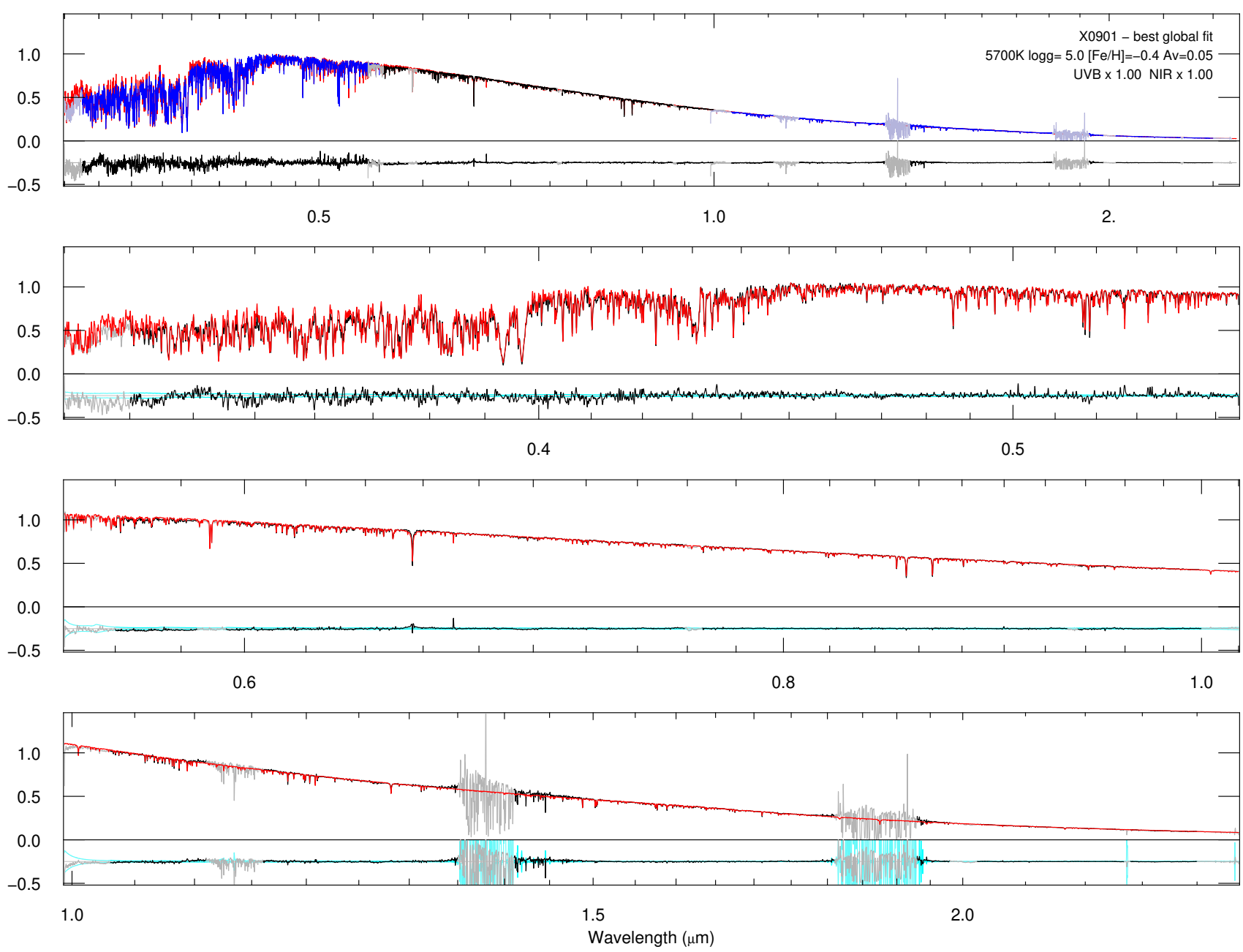

Fig. A.1. Best fit to the XSL spectrum of HD 111515 (observation X0901), at $R=3000$, using all (but masked) wavelengths. Each XSL-arm is rescaled independently to optimize the global quality of the fit. The model is shown in red. In the top panel, the blue-black-blue color-scheme identifies the three arms of X-shooter spectra, which are then shown separately (in black) in the three lower panels. Masked wavelength regions in the XSL data are plotted in gray. The noise spectra from the XSL pipeline are in cyan. 

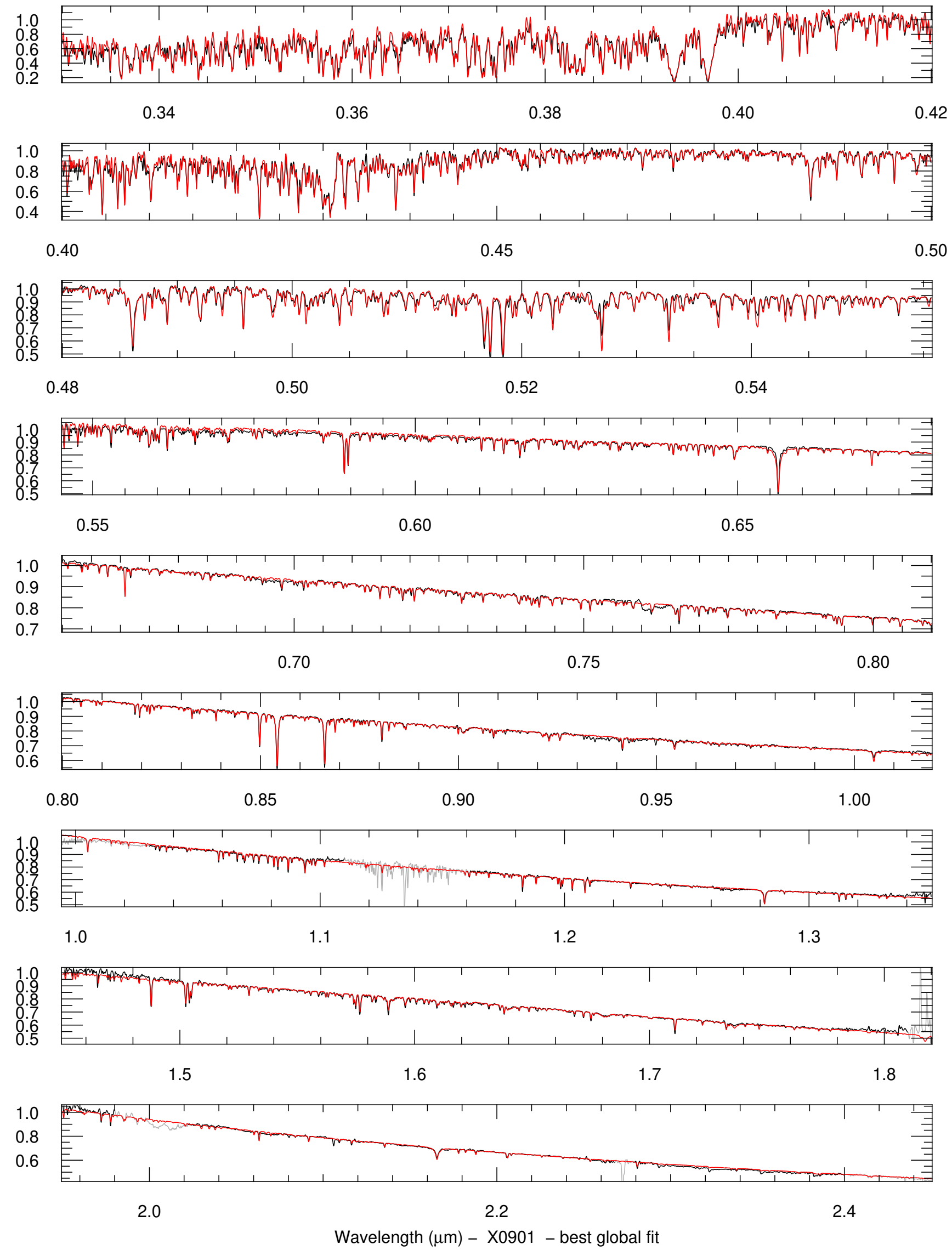

Fig. A.2. Zooms into various wavelength regions, for the match shown in Fig. A.1 (HD 111515, observation X0901), at $R=3000$. The model is shown in red. Masked wavelength regions in the XSL data are plotted in gray. 
A. Lançon et al.: A comparison between X-shooter spectra and PHOENIX models across the HR-diagram
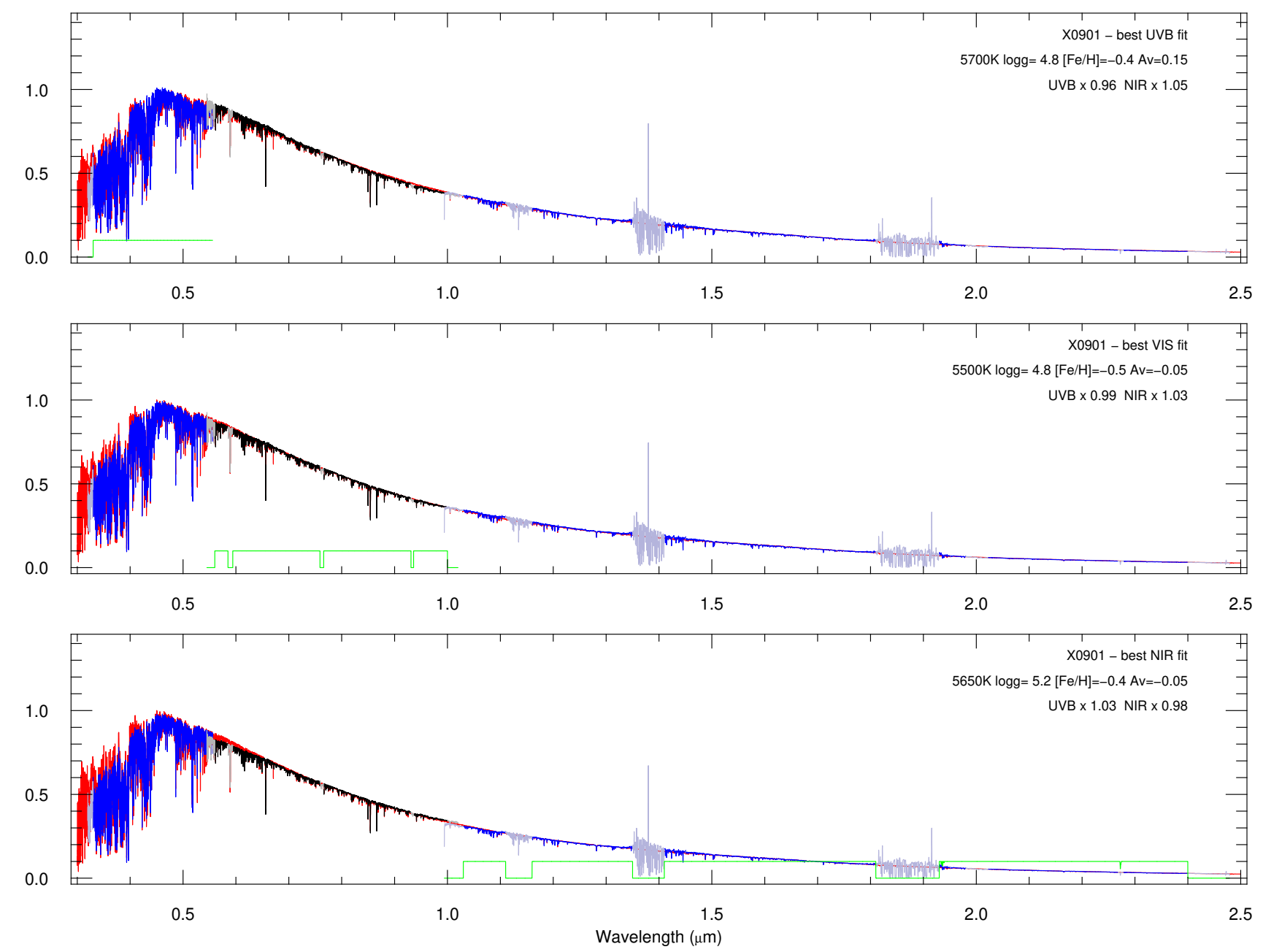

Fig. A.3. Best fits to the XSL spectrum of HD 111515 (observation X0901), but this time using only one of the three XSL arms in the optimization process: the UVB arm in the top panel, the VIS arm in the middle, and the NIR arm in the lower panel. The best-fit synthetic spectrum is shown in red, the empirical spectra in blue and black, or in gray for the masked regions (the mask, rescaled for display purposes, is displayed in green). In each panel, the arm-spectra not used in the fit are simply overlaid, after independent rescaling to the level of the synthetic spectrum. The scaling factors in the legends are those applied to the UVB and NIR observations relative to the VIS observations. The parameters vary between the panels. 




Fig. A.4. $\chi^{2}$ distributions around the minimum, as a function of the wavelength range used to constrain the fits and as a function of the four model parameters (here, $[\alpha / \mathrm{Fe}]=0$ is assumed). The $\chi^{2}$ values are rescaled to a minimum of 1 for display; only values smaller than 1.4 are shown. For this star, the parameter estimates based on each arm separately or on all arms jointly are consistent with each other. The bottom left panel illustrates effects of the discrete sampling of the model grid: at $5700 \mathrm{~K}$, the available values of the other parameters lead to a higher global $\chi^{2}$ than at 5600 or $5800 \mathrm{~K}$. To produce Figs. A.1, A.2, and A.3 we have interpolated in the original model grid around the minima of the present figure - hence the slightly different parameters in those figures.

\section{Appendix B: Differences between neighboring models}

As a reference for the interpretation of Fig. 2, we show here how the finite sampling of the original grid of theoretical spectra may affect $D$. To this purpose, we have compared models chosen to lie close to XSL observations, with models one grid-step away in $T_{\mathrm{eff}}, \log (g)$, or $[\mathrm{Fe} / \mathrm{H}]$, and we have produced Fig. B.1 with the largest $D$ among these. In the left panels, the model grid is used as it is originally, and in the right panels the grid is interpolated as described in Sect. 4.1.

Among $T_{\text {eff }}, \log (g)$, and $[\mathrm{Fe} / \mathrm{H}]$, the single parameter producing the largest difference $D$ over one grid step of the original grid is $\log (g)$ for hot stars (via hydrogen absorption features, in particular the shape of the Balmer lines and of the Balmer jump; triangular symbols), and $[\mathrm{Fe} / \mathrm{H}]$ for most of the red giant branch (small disks). Interpolation between models increases the significance of the trends discussed in Sect. 5.1 and 5.2, without changing the nature of the trends. 

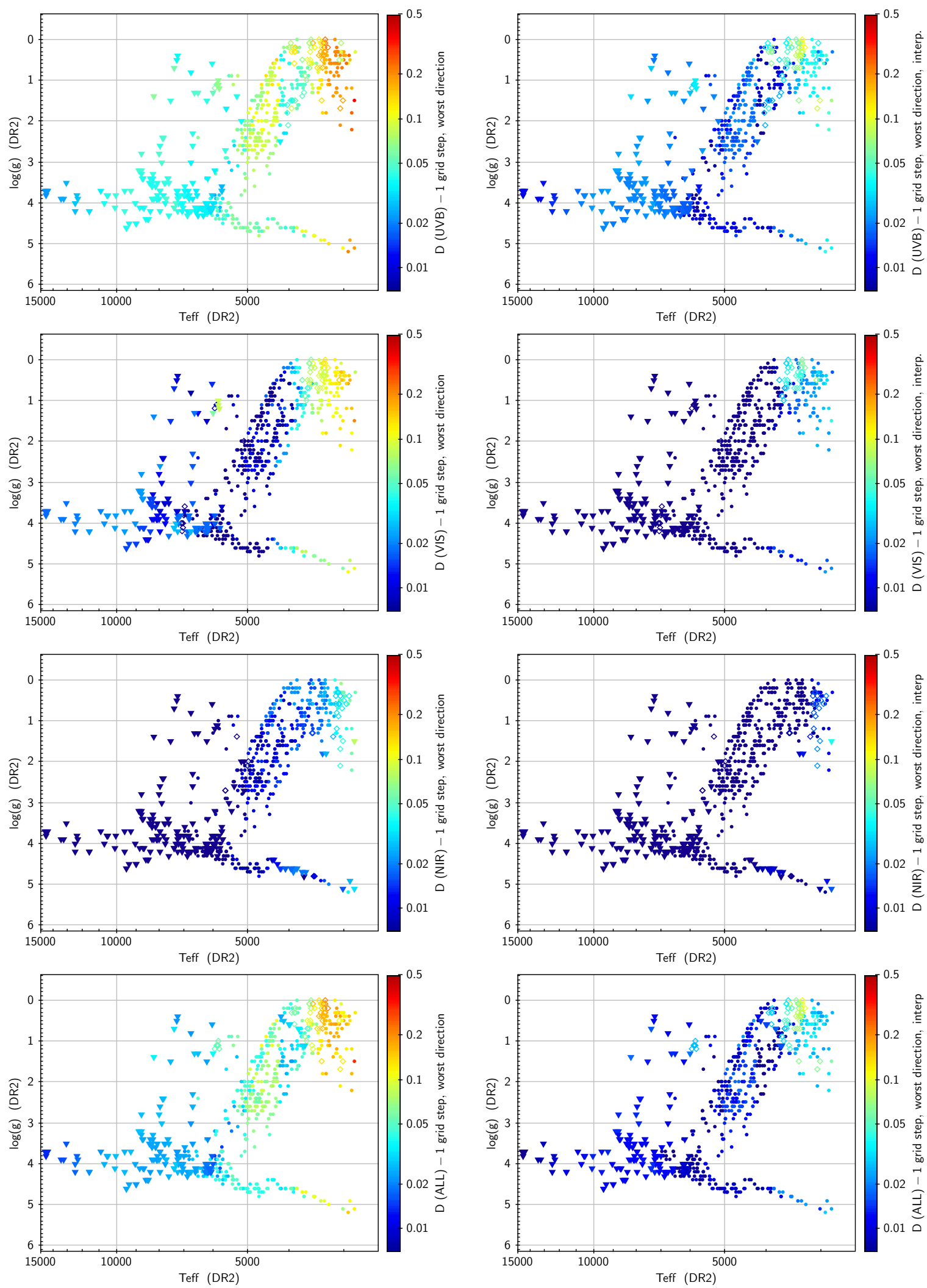

Fig. B.1. Sensitivity of $D$ (Eq. (1)), measured at $R=500$, to one step in $T_{\text {eff }}, \log (g)$ or $[\mathrm{Fe} / \mathrm{H}]$ in the worst direction in the grid of theoretical spectra. One symbol is plotted for each XSL spectrum. It is positioned according to the parameters of Arentsen et al. (2019) (as elsewhere in this article), and colored according to the largest 1-step difference $D$ between the model closest to the XSL-spectrum's parameters on one hand, and the six models surrounding that one on the other. In the left panels, the original grid of GSL models is used, while in the right panels interpolation was used to reduce the grid steps as detailed in Sect. 4.1. Triangles, small disks, or open diamonds indicate, respectively, that the largest $D$ between neighboring models in the grid corresponds to a change in $\log (g)$, in $[\mathrm{Fe} / \mathrm{H}]$ or in $T_{\text {eff }}$. From top to bottom, the wavelength ranges used are the UVB, the VIS, and the NIR arms, and finally all X-shooter wavelengths (ALL). 


\section{Appendix C: Direct XSL-GSL comparisons with the parameters from DR2}



Fig. C.1. Comparison of the SED of HD 147550 (observation X0245) with the (interpolated) GSL model nearest the parameters of DR2 (Arentsen et al. 2019), at $R=500$. In the uppermost panel, XSL data are shown in blue, black, and blue for the UVB, VIS, and NIR arms, over the model in red. In the lower panels, the model in red is overlaid on data in black. At this resolution the main disagreements are around the ends of the Hydrogen absorption line series, in particular around $370 \mathrm{~nm}$. With $A_{\mathrm{V}}=0.25$, the extinction law of Cardelli et al. (1989) with $R_{\mathrm{V}}=3.1$ behaves adequately.

In this section, only $A_{\mathrm{V}}$ is a fitted parameter; other parameters are taken from Arentsen et al. (2019). We present examples in order of decreasing effective temperature. To be readable in small format, plots are all at $R=500$.

As discussed in Sect. 5.1, models and observations agree well above $5000 \mathrm{~K}$, with only few exceptions. Figure C.1 illustrates the excellent match obtained for the hot main sequence star HD 147550 with the parameters of Arentsen et al. (2019) (X0245; $\left.T_{\text {eff }}=10000 \mathrm{~K}\right)$. The dip in the model shortwards of the Balmer series is a known peculiarity of the GSL library. When zooming into the figure (or at higher spectral resolution), one can see that the metal lines in the XSL spectra are on average stronger than in the synthetic spectrum, and also that the relative central depths of low- and high-order hydrogen lines in the Paschen and Brackett series are not precisely reproduced, even though the Balmer lines and the Balmer jump are modeled well. An example of a comparatively poor match to a warm star is given in Fig. C.2: the slope at the blue end of the NIR arm is slightly off. Taken individually, this offset would be consistent with flux calibration errors; but an offset in the other direction is seen for only 2 out of 21 warm stars in XSL. Nevertheless, the average offset is small compared to the dispersion between objects and we do not discuss it further.

For solar analogs, the fits are also satisfactory, with some discrepancies appearing (at $R=500$ ) mostly in UVB features sensitive to abundances of light elements or $\alpha$-elements. An example is given in Fig. C.3 for HD 55693 (X0585; $T_{\text {eff }}=5750 \mathrm{~K}$, $\log (g)=4.2,[\mathrm{Fe} / \mathrm{H}]=0.2)$. We note that the best-fit model in GSL (not shown) has as somewhat higher $T_{\text {eff }}$, of $6000 \mathrm{~K}$. An example at subsolar metallicity $([\mathrm{Fe} / \mathrm{H}]=-1.5)$, for which $[\alpha / \mathrm{Fe}]=+0.4$ improves the match from good to excellent, is provided in Fig. C.4 with star G187-40 (X0198).

Below $5000 \mathrm{~K}$, the agreement between models and observations is progressively lost in a systematic way. At low metallicity,


Fig. C.2. Same as Fig. C.1, but for HD 167946 (observation X0460). Wavelengths below $1.03 \mu \mathrm{m}$ are affected by large flux calibration errors. Between 1.03 and $1.1 \mu \mathrm{m}$ the situation is unclear: while flux calibration errors may be responsible for some of the discrepancy seen for this spectrum, we note that a similar discrepancy is seen in most hot stars (out of 21 XSL spectra between 9000 and $13000 \mathrm{~K}$, only two have fluxes above those of the model in that region), while the discrepancy tends to be the other way round in cool stars (empirical fluxes higher than the model fluxes).

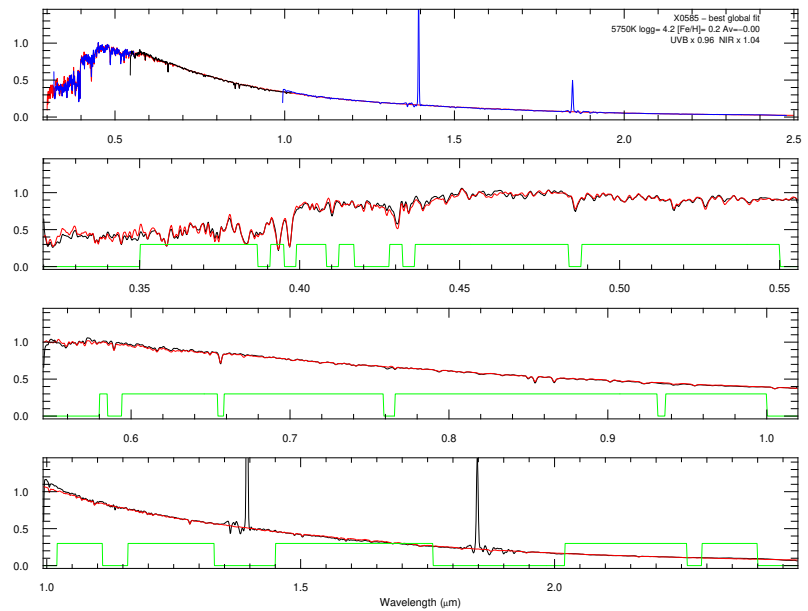

Fig. C.3. Comparison of the SED of the solar analog HD 55693 (observation X0585) with the (interpolated) GSL model nearest the parameters of Arentsen et al. (2019), at $R=500$. We note that a slightly higher $T_{\text {eff }}$ would provide an even better match.

discrepancies are obvious to the eye even when all wavelengths are used to optimize $A_{\mathrm{V}}$, as in Fig. C.5: the observed energy distributions are more peaked around $550 \mathrm{~nm}$ than the models, and the choice to rescale each XSL arm-spectrum independently to the local level of the model leads to systematic discontinuities at $1 \mu \mathrm{m}$. The discrepancies are even more striking when the best $A_{\mathrm{V}}$ is determined from one of the spectral arms only (Figs. C.6, C.7 and C.8 for estimates of $A_{\mathrm{V}}$ based on the UVB, the VIS, and the NIR ranges only). It is noteworthy that the attempt to match UVB observations and models (with the parameters of Arentsen et al. 2019) leads to positive $A_{\mathrm{V}}$ in all cases, and consequently also when all wavelengths are exploited. In general, the value of the preferred $A_{\mathrm{V}}$ decreases with wavelength.

Still in the range between 4000 and $5000 \mathrm{~K}$, the comparisons of the empirical and theoretical energy distributions for 
A. Lançon et al.: A comparison between X-shooter spectra and PHOENIX models across the HR-diagram

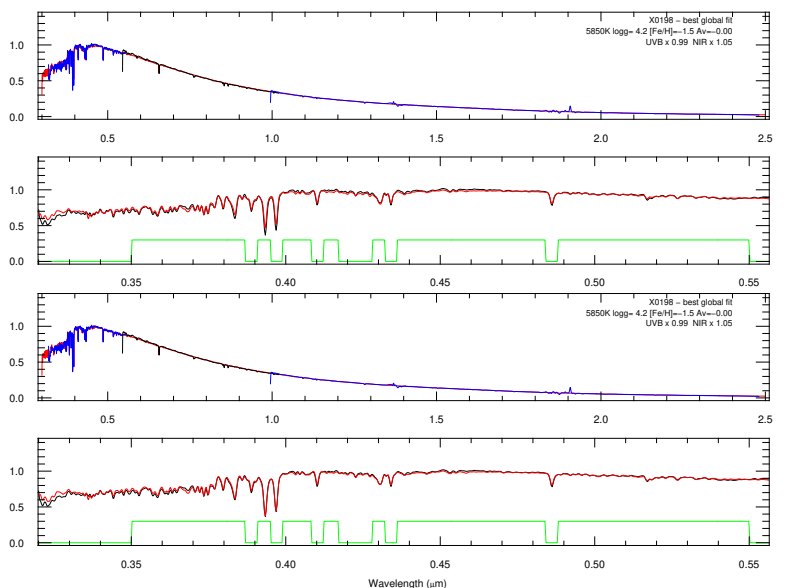

Fig. C.4. Illustration of the small effects of a change of 0.4 in $[\alpha / \mathrm{Fe}]$ at $R=500$, using the low metallicity near-solar star G187-40 (observation X0198) with the parameters of Arentsen et al. (2019). The top two panels show that nearest model at $[\alpha / \mathrm{Fe}]$ of 0 , the bottom two panels at $[\alpha / \mathrm{Fe}]=+0.4$

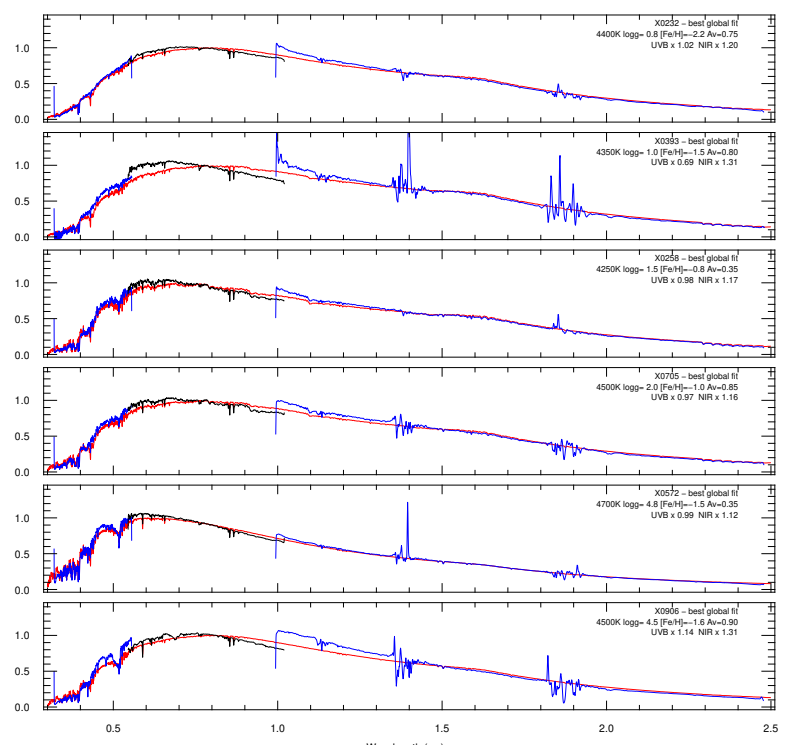

Fig. C.5. Typical comparisons between XSL and GSL energy distributions for low metallicity stars with estimated $T_{\text {eff }}$ between 4000 and $5000 \mathrm{~K}$, at $R=500$, when adopting the parameters of Arentsen et al. (2019) unchanged. Gravity increases from top to bottom: HD 165195 (X0232), HD 1638 (X0258), NGC 68381037 (X0705), LHS 1841 (X0572), LHS 343 (X0906). The discontinuities between the UVB, VIS and NIR segments of the XSL data appear when the general energy distribution is not well matched by the models, because our method scales each arm independently: no other $A_{\mathrm{V}}+$ scaling combination would provide a smaller discrepancy $D$ (Eq. (1)) across all wavelengths. In other words, $D$ would be worse if the UVB, VIS and NIR segments were first merged and $A_{\mathrm{V}}$ was estimated subsequently.

stars with solar-like metallicities (Fig. C.9) is, in fact, generally slightly better than at subsolar metallicities. The outlier in the top panel of that figure is a red supergiant for which the DR2parameters seem far off, possibly as a result of variability; other red supergiants are not this drastically discrepant. The other panels are representative. Although figures with $A_{\mathrm{V}}$ determined from single arms display trends similar in nature to those shown previously at lower metallicity, the discrepancies have a somewhat smaller amplitude, on average (only two of these figures are shown, in Figs. C.10 and C.11).

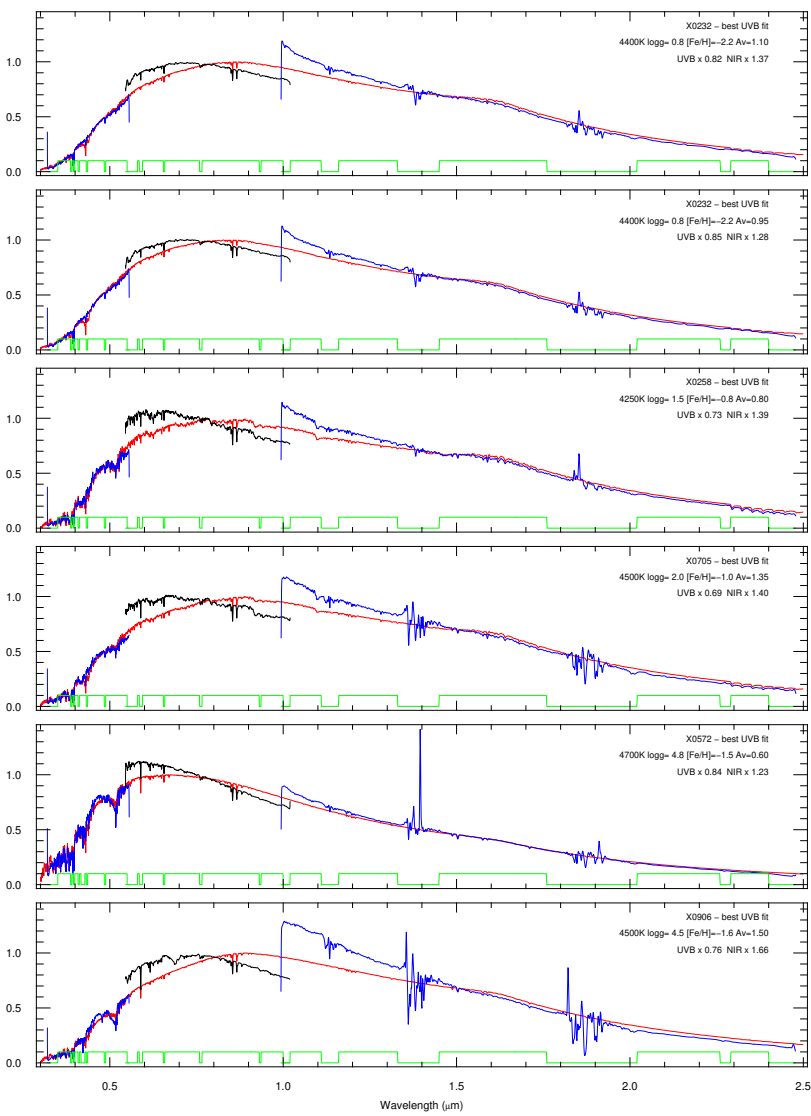

Fig. C.6. Same as Fig. C.5, except that $A_{\mathrm{V}}$ is estimated using only UVB data.

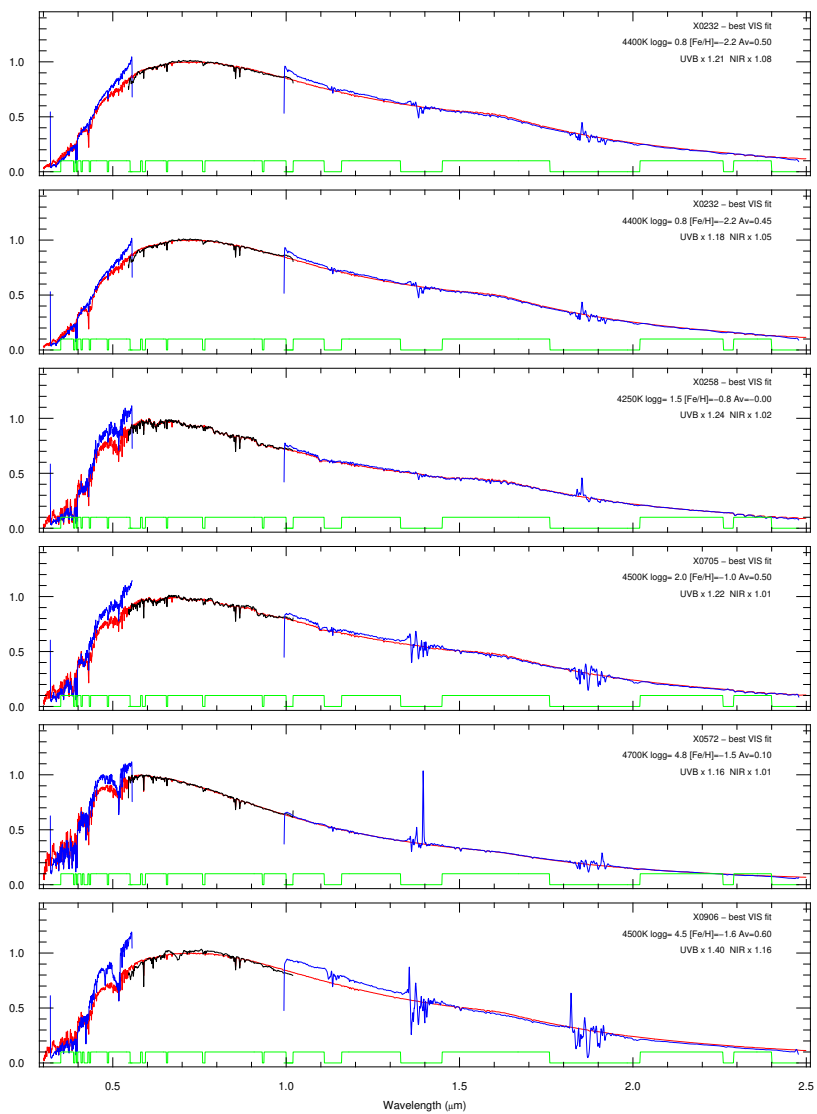

Fig. C.7. Same as Fig. C.5, except that $A_{\mathrm{V}}$ is estimated using only VIS data.

A97, page 25 of 31 

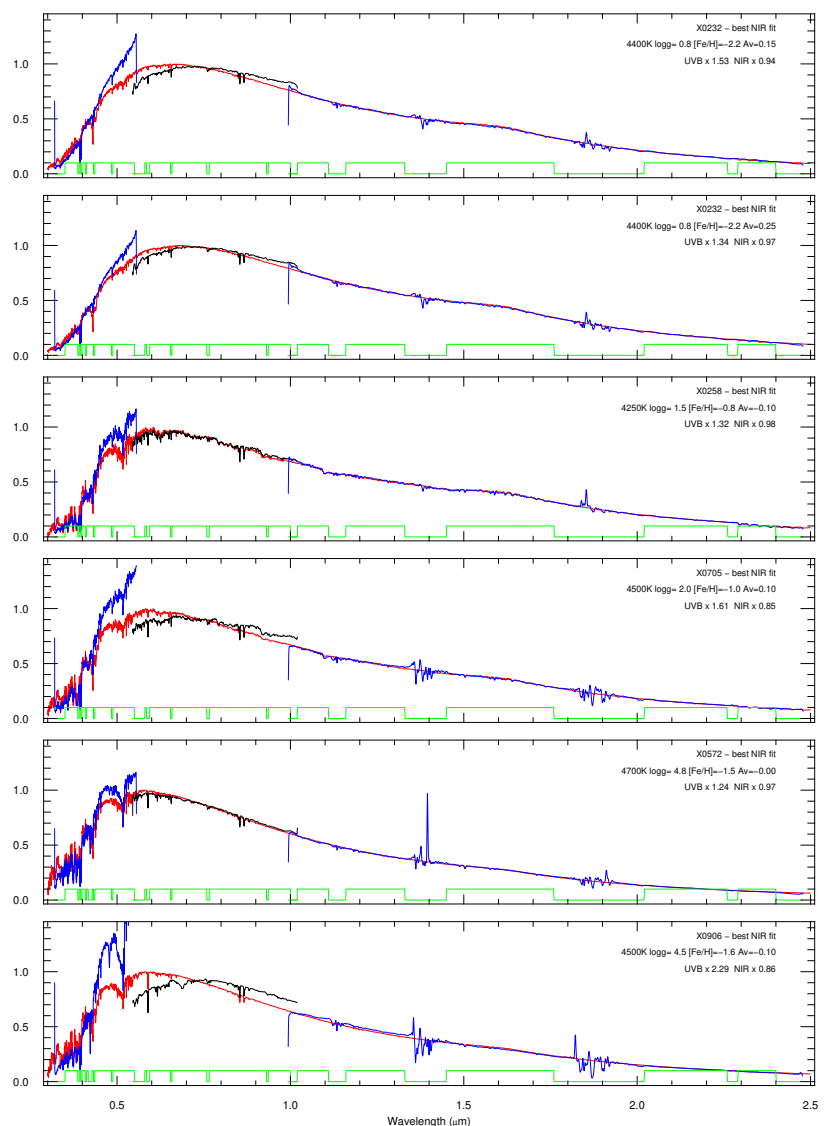

Fig. C.8. Same as Fig. C.5, except that $A_{\mathrm{V}}$ is estimated using only NIR data.

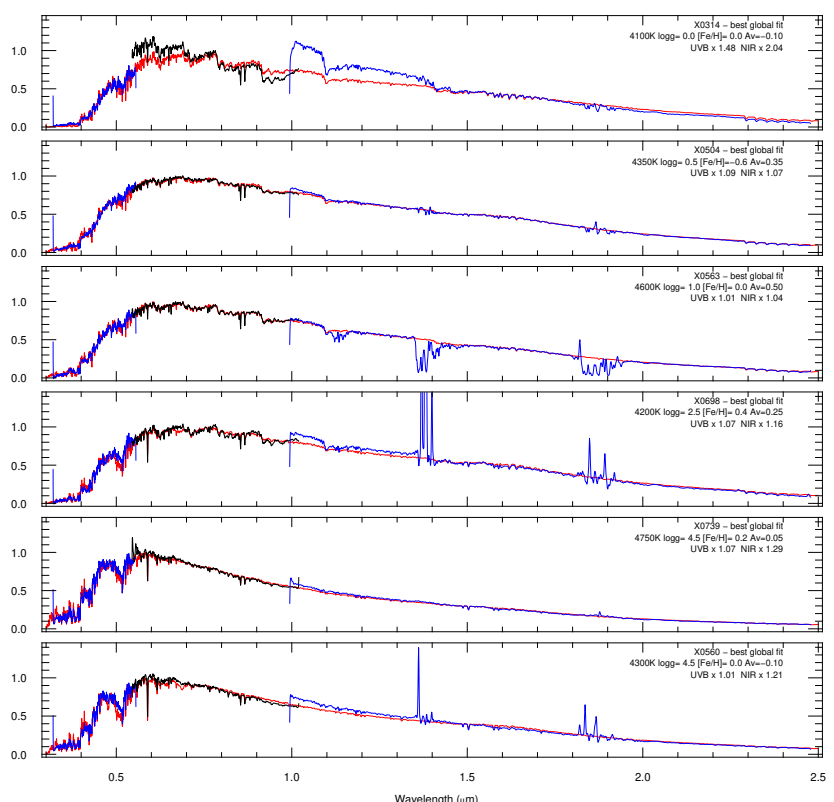

Fig. C.9. Typical comparisons between XSL and GSL energy distributions for stars with relatively high metallicities and with estimated $T_{\text {eff }}$ between 4000 and $5000 \mathrm{~K}$, at $R=500$, when adopting the parameters of Arentsen et al. (2019) unchanged. Gravity increases from top to bottom: HD 50877 (X0314), BBB SMC 104 (X0504), HD 44391 (X0563), 2MASS J18351420-3438060 (X0698), HD 218566 (X0739), HD 21197 (X0560). As in Fig. C.5, discontinuities between the UVB, VIS, and NIR segments of the XSL data appear when the general energy distribution is not well matched by the models.

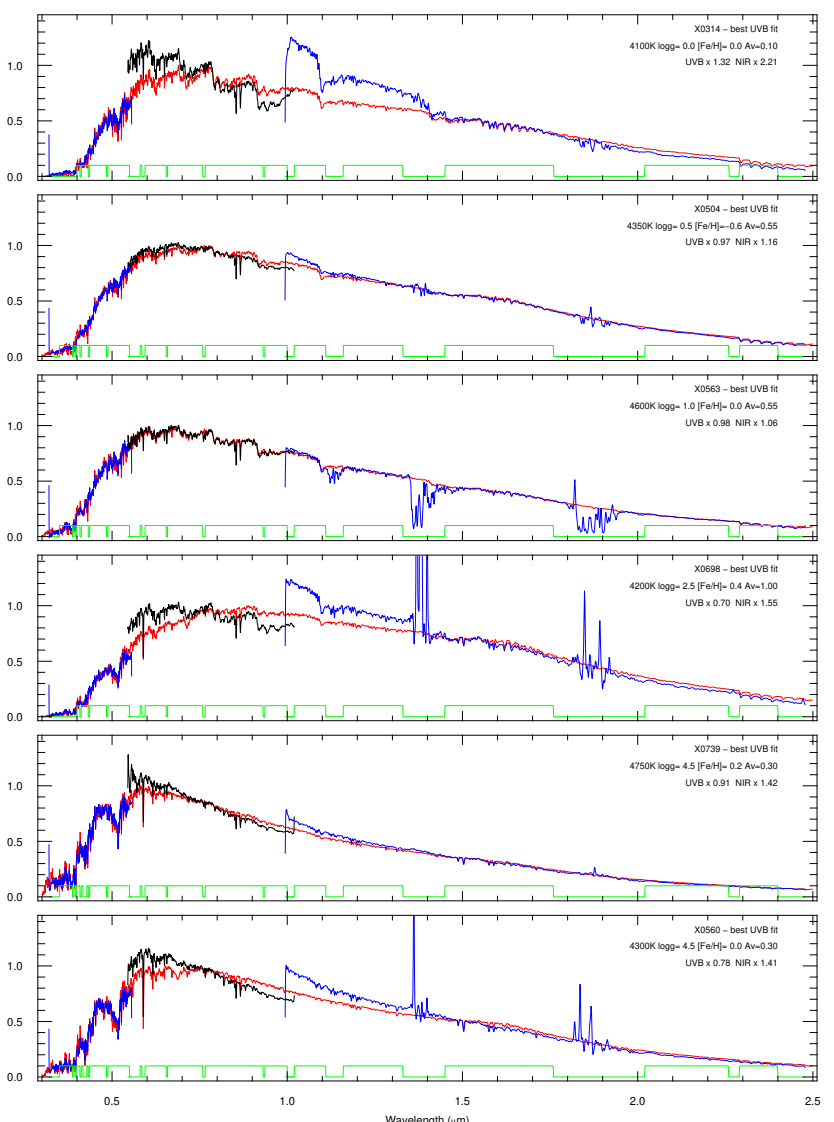

Fig. C.10. Same as Fig. C.9, except that $A_{\mathrm{V}}$ is estimated using only UVB data.



Fig. C.11. Same as Fig. C.9, except that $A_{\mathrm{V}}$ is estimated using only VIS data. 


\section{Appendix D: Improvements obtained when replacing DR2 parameters with best fit parameters.}

In Fig. 4, we showed how a free choice of best-fit parameters improves the goodness-of-fit measure $D$, in the case of a fit to all wavelengths. Here, Figs. D.1 and D.2 provide this information for the four variants of the fits: UVB only, VIS only, NIR only or ALL wavelengths.
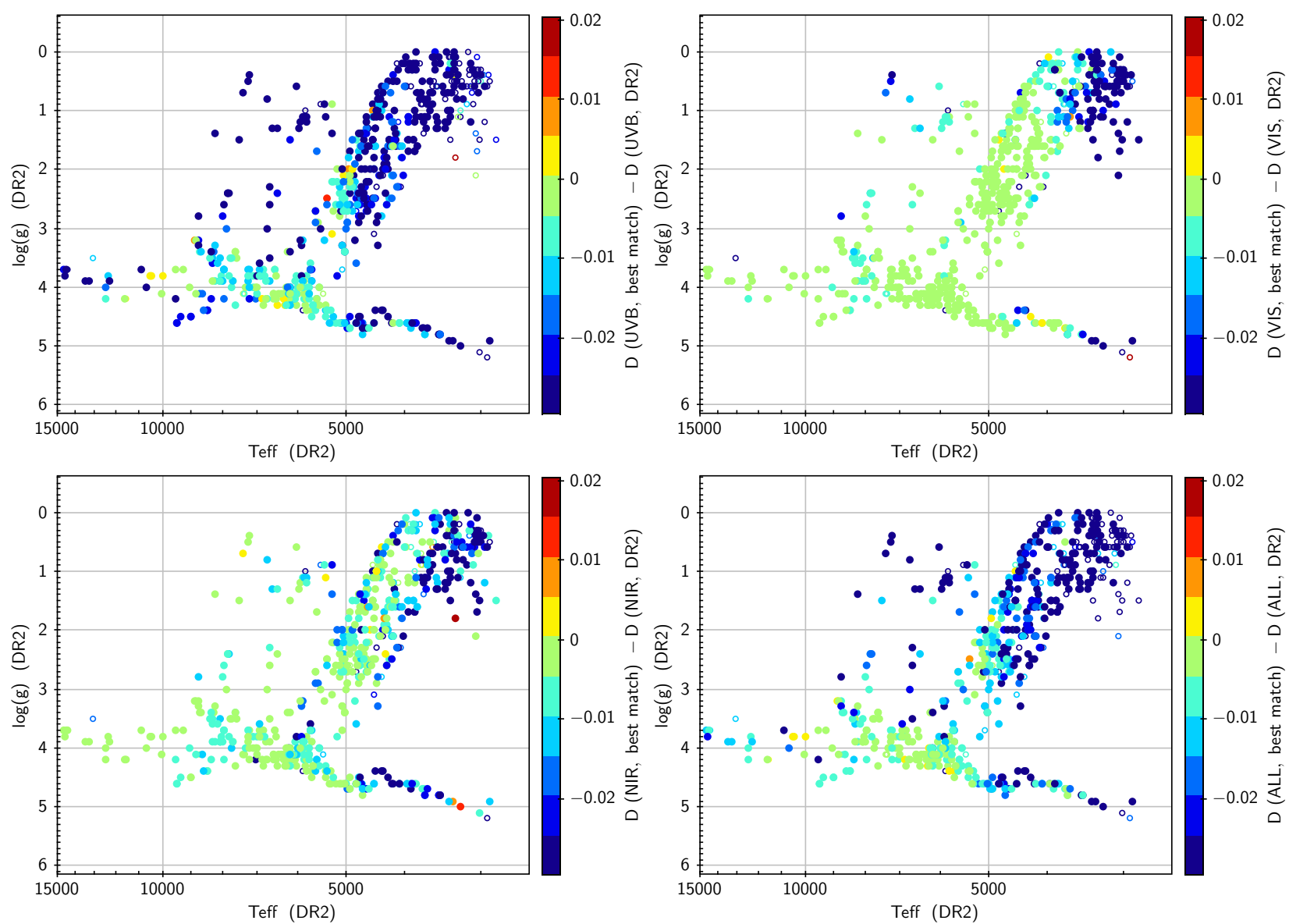

Fig. D.1. Improvement in the XSL-GSL match when instead of adopting stellar parameters from Arentsen et al. (2019), the parameters are optimized. Only the bottom right panel was shown in the main body of the paper (Fig. 4). 

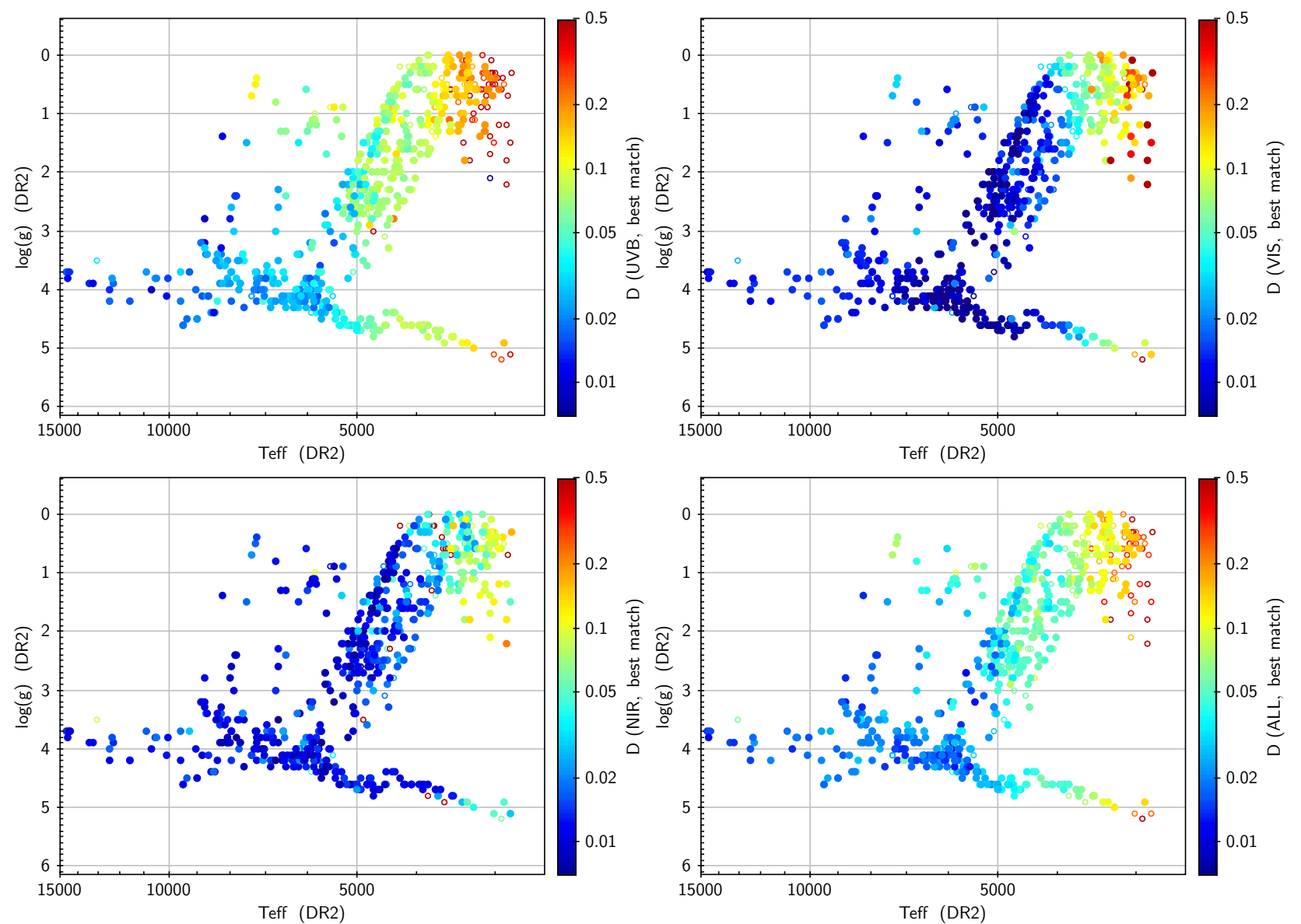

Fig. D.2. Discrepancy measure $D$ between the XSL and GSL spectra, as obtained for the best-match synthetic spectra. The four panels correspond best-fit determinations and calculations of $D$ based respectively on the UVB, VIS, and NIR arm of X-shooter, and on the three arms combined. To be compared to Fig. 2, where the stellar parameters of XSL-DR2 were assumed. 


\section{Appendix E: Parameter estimates : differences between arms}
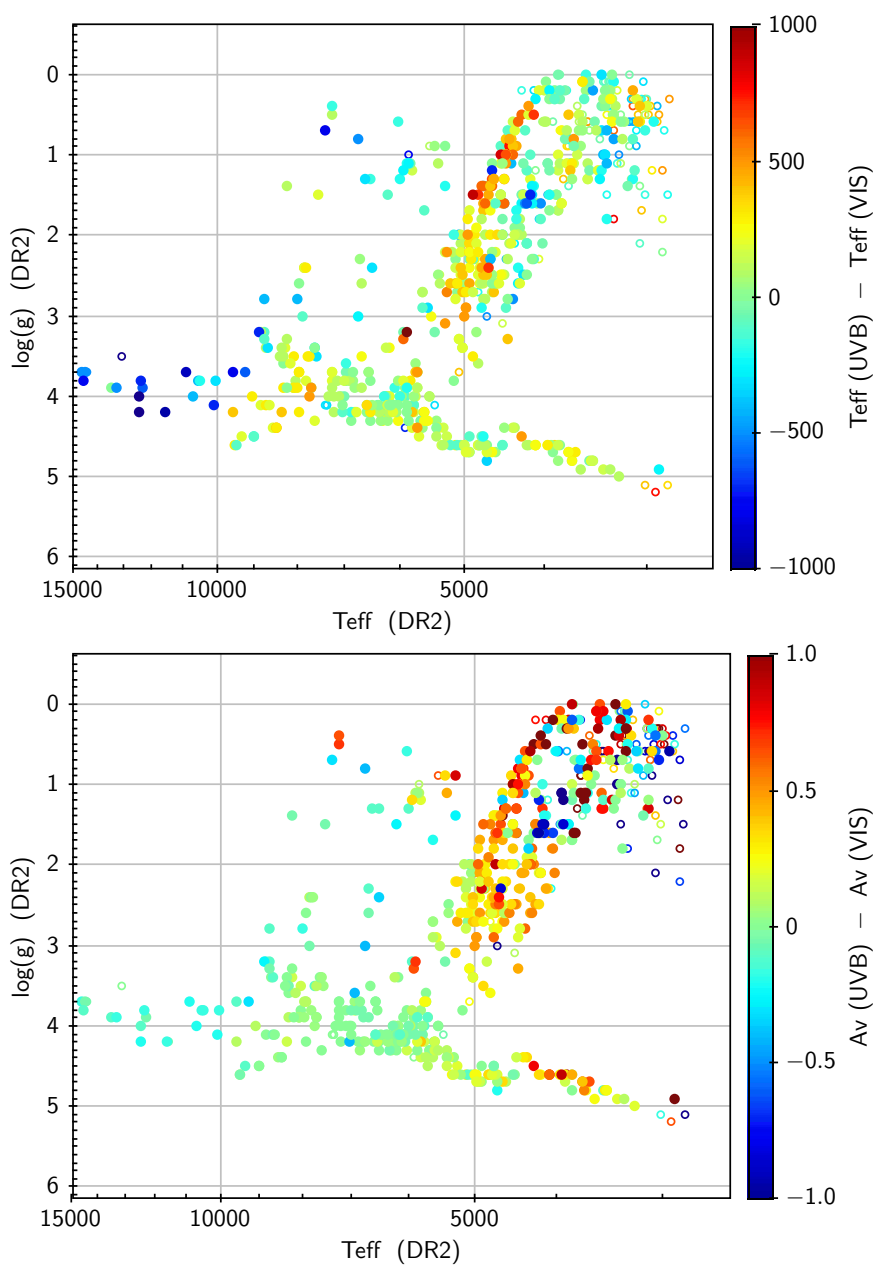

Fig. E.1. HR diagrams colored by $\Delta T_{\text {eff }} \equiv T_{\text {eff }}(\mathrm{UVB})-T_{\text {eff }}$ (VIS) (top) and by $\Delta A_{\mathrm{V}} \equiv A_{\mathrm{V}}(\mathrm{UVB})-A_{\mathrm{V}}$ (VIS) (bottom), based on inverse-variance weighted $\chi^{2}$ comparisons at $R=500$.

As discussed in Sect. 5.2.1, differences between the parameters derived separately from the three X-shooter arms are an indication of imperfection in the models, when they are systematic (otherwise they may result from flux calibration errors or other anecdotical artifacts). The main trends have been discussed in the main text. A few more localized systematics are mentioned here.

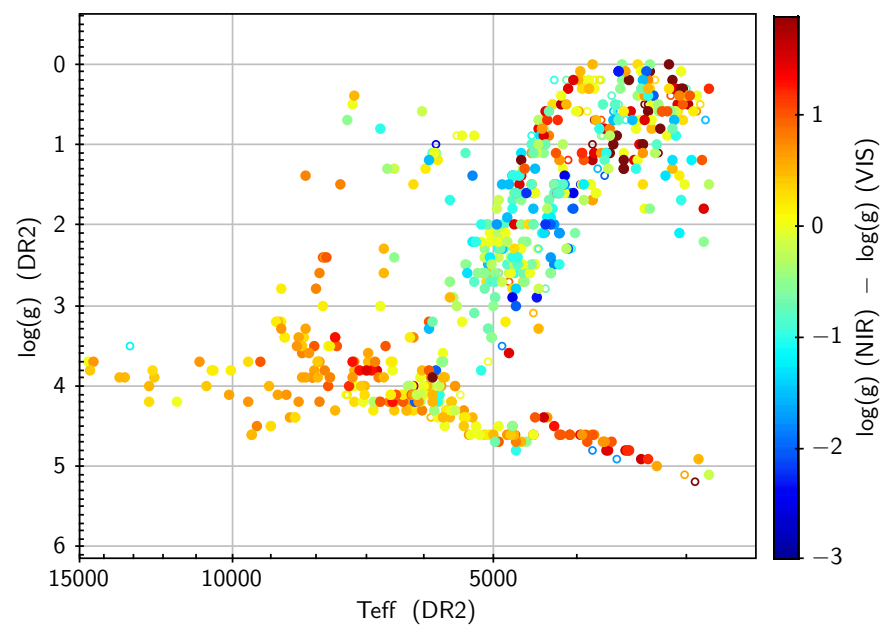

Fig. E.2. HR diagram colored by $\log (g)(\mathrm{NIR})-\log (g)$ (VIS), based on inverse-variance weighted $\chi^{2}$ comparisons at $R=500$.

A first example is the difference $\Delta T_{\text {eff }} \equiv T_{\text {eff }}(\mathrm{UVB})-$ $T_{\text {eff }}$ (VIS), which correlates with $\Delta A_{\mathrm{V}} \equiv A_{\mathrm{V}}(\mathrm{UVB})-A_{\mathrm{V}}(\mathrm{VIS})$ on the whole, but shows a more discriminant behavior in the HR diagram. For instance, Fig. E.1 highlights that while the majority of giants between 4000 and $5000 \mathrm{~K}$ lead to a positive $\Delta A_{\mathrm{V}}$, a smaller fraction has a positive $\Delta T_{\text {eff }}$. Those are typically the lowmetallicity giants: $90 \%$ of the giant branch stars with $[\mathrm{Fe} / \mathrm{H}]<$ -1.5 (according to Arentsen et al. 2019) have best-fit temperatures with $\Delta T_{\text {eff }} \geqslant 100 \mathrm{~K}$, while this fraction is about $45 \%$ for more metal-rich giants between 3800 and $7000 \mathrm{~K}$ (or also for the whole sample of spectra). The median $\Delta T_{\text {eff }}$ for metal-poor giants is $\sim 400 \mathrm{~K}$. These numbers remain the same whether one assumes $[\alpha / \mathrm{Fe}]=0$ or $[\alpha / \mathrm{Fe}]=+0.4$. On the main sequence at temperatures between 10000 and $15000 \mathrm{~K}$ (20 stars), $\Delta T_{\text {eff }}$ is smallest : it is systematically negative with typical values near $-600 \mathrm{~K}$. Extinction is not involved here, since these stars are in general reddened very little.

In Fig. E.2, the HR diagram is colored by the difference between arms that shows the strongest local systematics when we require that the NIR arm be involved. This turns out to be the difference between the surface gravities derived from the NIR and from another arm. In the comparison with the GSL models, the NIR arm pulls towards high gravities on the main sequence, while it pulls towards low gravities for many intermediate luminosity red giants. 


\section{Appendix F: Effects of $[\alpha / \mathrm{Fe}]$ on parameters estimated here}
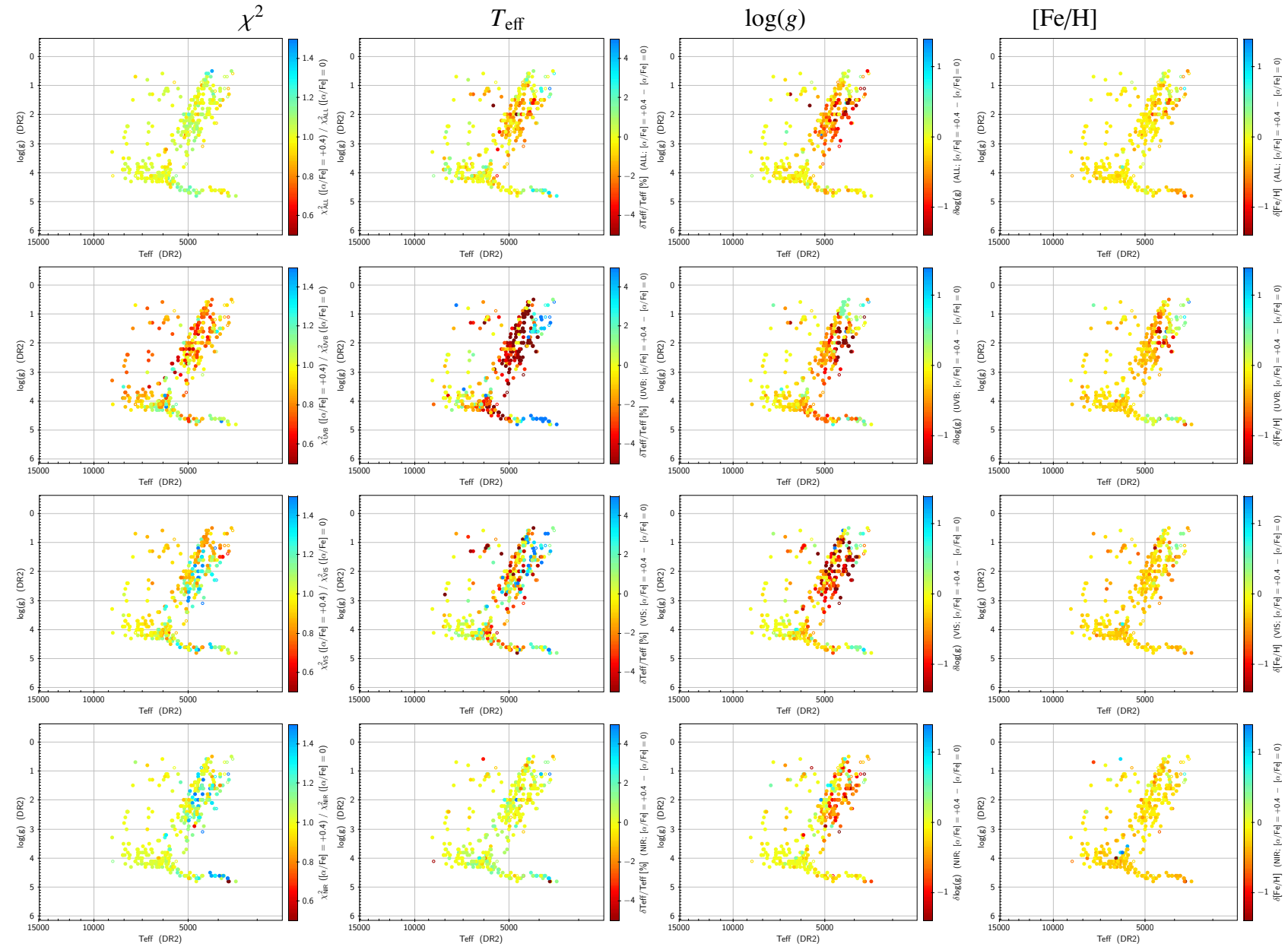

Fig. F.1. Effects of a switch from models at $[\alpha / \mathrm{Fe}]=0$ to models at $[\alpha / \mathrm{Fe}]=+0.4$. The model-data comparison is carried out at $R=3000$, with interpolation in the model grid, using the inverse-variance weighted $\chi^{2}$. The restricted sample shown in these figures corresponds to DR2-parameters in the range for which models are available at $[\alpha / \mathrm{Fe}]=+0.4$.

Figure F.1 summarizes the effect of a change in the assumed $[\alpha / \mathrm{Fe}]$ on the best-fit parameters from the comparison of the XSL spectra, at $R=3000$, with the PHOENIX models from Husser et al. (2013), using the inverse-variance weighted $\chi^{2}$ over different wavelength ranges. Two values of $[\alpha / \mathrm{Fe}]$ are considered: 0 and +0.4 .

The leftmost column shows the ratio $\chi^{2}(0) / \chi^{2}(+0.4)$. The fit to the whole energy distribution and the fit to the NIR arm are not very sensitive to $[\alpha / \mathrm{Fe}]$, but if anything would mildly favor the solar ratio (see Table F.1). The fit to the UVB on the other hand favors a super-solar $[\alpha / \mathrm{Fe}]$ everywhere except on the lower main sequence, and a comparison with the metallicity distribution in Fig. 1 shows this to be essentially independent of metallicity. The anticorrelation between $[\alpha / \mathrm{Fe}]$ and $[\mathrm{Fe} / \mathrm{H}]$ characteristic of the Milky Way is seen at a significant level only in the fit to VIS wavelengths. In the main text of this paper, the criterion adopted for saying that a spectrum "prefers $[\alpha / \mathrm{Fe}]=+0.4$ " is that it reduces the $\chi^{2}$ in the UVB without degrading the $\chi^{2}$ in the VIS or NIR ranges, which in effect confers the VIS an important role. The resulting subset is shown in Fig. F.2.

The change from solar to super-solar $[\alpha / \mathrm{Fe}]$ leads, in most areas of the HR diagram, to a decrease in $T_{\text {eff }}$, a decrease in $\log (g)$ and a decrease in metallicity for a given XSL spectrum.
Table F.1. Fractions of spectra that prefer $[\alpha / \mathrm{Fe}]=+0.4$, for metal-poor and metal-rich stars.

\begin{tabular}{ccc}
\hline \hline $\begin{array}{c}\text { Wavelength } \\
\text { range }\end{array}$ & {$[\mathrm{Fe} / \mathrm{H}]<-0.5$} & {$[\mathrm{Fe} / \mathrm{H}] \geqslant-0.5$} \\
\hline UVB & $90 \%$ & $70 \%$ \\
VIS & $90 \%$ & $46 \%$ \\
NIR & $50 \%$ & $35 \%$ \\
ALL & $35 \%$ & $42 \%$ \\
\hline
\end{tabular}

Notes. Fits are done at $R=3000$, using the inverse-variance weighted $\chi^{2}$.

The decrease in $[\mathrm{Fe} / \mathrm{H}]$ logically compensates the increase in total metallicity $[\mathrm{M} / \mathrm{H}]$ in the $\alpha$-enhanced models (the $\alpha$ elements being added at a given $[\mathrm{Fe} / \mathrm{H}])$. The systematic changes in $T_{\text {eff }}$ and $\log (g)$ occur in addition to that partial compensation. The trends are, in general, seen whether one fits the UVB, the VIS or the NIR wavelength ranges, with only few exceptions. The trend towards lower $T_{\text {eff }}$ at higher $[\alpha / \mathrm{Fe}]$ is not seen in the NIR range, although that wavelength range by itself recovers the changes in $\log (g)$ or $[\mathrm{Fe} / \mathrm{H}]$ with $[\alpha / \mathrm{Fe}]$, found in the other spectral ranges. 
A. Lançon et al.: A comparison between X-shooter spectra and PHOENIX models across the HR-diagram

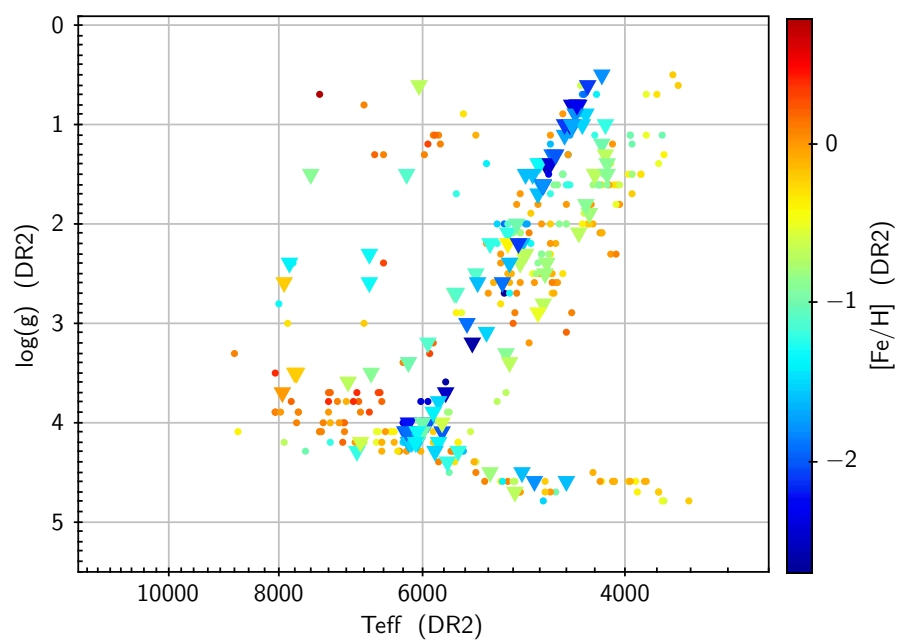

Fig. F.2. Large triangles: stars classified in this paper as favoring models with $[\alpha / \mathrm{Fe}]=+0.4$ over solar-scaled models, in model-data comparisons based on the inverse-variance $\chi^{2}$ in which parameters are free. They have the following XSL identifiers: X0007, X0023, X0024, X0065, X0070, X0072, X0075, X0077, X0089, X0092, X0122, X0123, X0137, X0140, X0143, X0151, X0157, X0171, X0176, X0198, X0200, X0206, X0213, X0225, X0232, X0238, X0282, X0286, X0290, X0350, X0352, X0359, X0361, X0366, X0380, X0388, X0389, X0392, X0394, X0408, X0415, X0417, X0418, X0419, X0432, X0442, X0445, X0446, X0449, X0464, X0477, X0480, X0490, X0496, X0500, X0521, X0522, X0536, X0547, X0548, X0549, X0566, X0598, X0600, X0601, X0604, X0611, X0613, X0618, X0619, X0621, X0623, X0627, X0630, X0634, X0640, X0643, X0670, X0678, X0681, X0682, X0687, X0700, X0702, X0728, X0733, X0734, X0757, X0770, X0775, X0827, X0843, X0848, X0856, X0857, X0863, X0866, X0867, X0870, X0878, X0882, X0884, X0886, X0893, X0897, X0899, X0900, X0906 